\title{
Note on the number of semistar operations, VIII
}

\author{
RYÛKI MATSUDA*
}

We concern with the number of star operations or of semistar operations on an integral domain or on a semigroup. Since all the proofs in [M 4,7,8] and [MS] are not necessarily complete, it is necessary for us to make a self-contained, well got together and systematically written paper on the subject. Some results in this paper are new. We confer Badawi-Houston [BH], Hedstrom-Houston [HH], Heinzer $[\mathrm{H}]$, and many other references.

Throughout the paper, $D$ denotes an integral domain, and $S$ denotes a nonzero g-monoid. A g-monoid means a submonoid of a torsion-free abelian additive group.

The set of star operations on $D$ is denoted by $\Sigma(D)$.

The set of star operations on $S$ is denoted by $\Sigma(S)$.

The set of semistar operations on $D$ is denoted by $\Sigma^{\prime}(D)$.

The set of semistar operations on $S$ is denoted by $\Sigma^{\prime}(S)$.

Let $T$ be an overring of $D$, and let $\mathrm{d}_{T}^{\prime}$ be the $\mathrm{d}^{\prime}$-operation on $T$. Then the descent of $\mathrm{d}_{T}^{\prime}$ to $D$ is denoted by $*_{T}$ (or by $*_{(T)}$ ).

Let $T$ be an oversemigroup of $S$, and let $\mathrm{d}_{T}^{\prime}$ be the $\mathrm{d}^{\prime}$-operation on $T$. Then the descent of $\mathrm{d}_{T}^{\prime}$ to $S$ is denoted by $*_{T}\left(\right.$ or by $\left.*_{(T)}\right)$.

Let $\Gamma$ be a totally ordered set, and let $X$ be a subset of $\Gamma$. Then ' inf ' of $X$ in $\Gamma$ is denoted by ' $\inf ^{\Gamma} X^{\prime}$ (or simply by ' $\inf X^{\prime}$ ). ' sup ' of $X$ in $\Gamma$ is denoted by ${ }^{\prime} \sup { }^{\Gamma} X^{\prime}$ (or simply by ' $\sup X^{\prime}$ ). Also inf ${ }^{\Gamma} X=-\infty$ means that $X$ is not bounded below, and inf ${ }^{\Gamma} X>-\infty$ means that $X$ is bounded below.

For any set $X$, the cardinality of $X$ is denoted by $|X|$.

Lemma 1. If $\left|\Sigma^{\prime}(S)\right|<\infty$, then the integral closure $\bar{S}$ of $S$ is a valuation semigroup.

Proof. Suppose the contrary. Let $G=\mathrm{q}(S)$. There exists an element $x \in G-\bar{S}$ such that $-x \notin \bar{S}$. Then we have $S\left[2^{n} x\right] \supsetneqq S\left[2^{n+1} x\right]$ for each positive integer $n$. Let $\mathrm{d}_{n}^{\prime}$ be the $\mathrm{d}^{\prime}$-operation on $S\left[2^{n} x\right]$, and let $*_{n}$ be the descent of $\mathrm{d}_{n}^{\prime}$ to $S$. There arises an infinite number of semistar operations $*_{1}, *_{2}, \cdots$ on $S$.

Received April 26, 2005; revised August 8, 2005.

2000 Mathematics Subject Classification. 13A15.

Key words. almost pseudo-valuation domain, Prüfer domain, pseudo-valuation domain, semigroup, star operation, semistar operation.

* Professor Emeritus, Ibaraki University, Mito, Ibaraki, 310-8512, Japan. 
Proposition 1. Let $V$ be a valuation semigroup with maximal ideal $M$.

(1) If $M$ is a principal ideal of $V$, then $|\Sigma(V)|=1$.

(2) If $M$ is not a principal ideal of $V$, then $|\Sigma(V)|=2$.

Proof. Let $v$ be a valuation on $G=\mathrm{q}(V)$ belonging to $V$, and let $\Gamma$ be the value group of $v$. Let $I$ be an ideal of $V$.

The case where there exists $x \in I$ such that $v(x)=\min v(I)$ : Then $I=(x)$.

The case where there exists $x \in V$ such that $v(x)=\inf { }^{\Gamma} v(I) \notin v(I)$ : Then $I=x+M$.

The case where inf ${ }^{\Gamma} v(I)$ does not exists: Then $I=I^{v}$.

(1) Each ideal of $V$ is divisorial. It follows that $\Sigma(V)=\{\mathrm{d}\}$.

(2) Since $V=M^{v}$, we have $\mathrm{v} \neq \mathrm{d}$. Let $*$ be a star operation on $V$. Then, if $M^{*}=M$, then $*=\mathrm{d}$, and if $M^{*}=V$, then $*=\mathrm{v}$.

The following Proposition is a ring version of Proposition 1.

Proposition 2. Let $V$ be a valuation domain with maximal ideal $M$.

(1) If $M$ is a principal ideal, then $|\Sigma(V)|=1$.

(2) If $M$ is not a principal ideal, then $|\Sigma(V)|=2$.

The set of nonzero fractional ideals of $D$ is denoted by $\mathrm{F}(D)$. The set of nonzero $D$-submodules of $K$ is denoted by $\mathrm{F}^{\prime}(D)$, where $K$ is the quotient field of $D$. The set of fractional ideals of $S$ is denoted by $\mathrm{F}(S)$. The set of $S$-submodules of $G$ is denoted by $\mathrm{F}^{\prime}(S)$, where $G$ is the quotient group of $S$.

Lemma 2. (1) Let $D$ be a valuation ring, or let $D$ be a Prüfer ring with exactly two maximal ideals $M$ and $N$ such that there exists a prime ideal $P$ of $D$ with $M \cap N \supsetneqq P \supsetneqq(0)$. Then $\mathrm{F}^{\prime}(D)=\mathrm{F}(D) \cup\{K\}$, where $K=\mathrm{q}(D)$.

(2) Let $S$ be a valuation semigroup. Then $\mathrm{F}^{\prime}(S)=\mathrm{F}(S) \cup\{G\}$, where $G=\mathrm{q}(S)$.

Hence each star operation on $D$ (resp. S) has a unique extension to a semistar operation on $D$ (resp. $S$ ).

Proof. (1) Assume that $D$ is not a valuation ring. Let $v$ (resp. $w$ ) be a valuation belonging to $V=D_{M}$ (resp. $\left.W=D_{N}\right)$. Let $I \in \mathrm{F}^{\prime}(D)$.

The case where $v(I)$ and $w(I)$ are bounded below: There exists a nonzero element $a \in D$ such that $a I \subset V$ and $a I \subset W$. Then $I \in \mathrm{F}(D)$.

The case where neither $v(I)$ nor $w(I)$ is bounded below: Let $x$ be a nonzero element of $K$. There exist elements $a \in D$ and $\pi \in P$ such that $x=\frac{a}{\pi}$. There exists an element $i \in I$ such that $v\left(i \pi^{2}\right)<0$. We have $\frac{1}{i \pi^{2}}=\frac{b}{s}$ for elements $b \in D$ and $s \in D-M$. It follows that $\frac{x}{i}=\frac{a b \pi}{s} \in V \cap W$. Hence $x \in i D \subset I$, and hence $I=K$.

The case where one of $v(I)$ and $w(I)$, say $w(I)$, is bounded below and the other is not bounded below: There exists an element $a \in D-\{0\}$ such that $w\left(\frac{1}{a}\right)<w(i)$ for each $i \in I$. Let $\pi \in P-\{0\}$, and choose $i_{0} \in I$ such that 
$v\left(i_{0}\right)<v\left(\frac{1}{a \pi}\right)$. Since $\frac{1}{a \pi i_{0}} \in V$, there exist $a \in D$ and $s \in D-M$ such that $\frac{1}{a \pi i_{0}}=\frac{d}{s}$. Then we have $w\left(i_{0}\right)<w\left(\frac{1}{a}\right) ;$ a contradiction.

Let $T$ be an overring of $D$, and let $*$ be a semistar operation on $T$. If we let $I^{\delta(*)}=(I T)^{*}$ for each $I \in \mathrm{F}^{\prime}(D)$, then $\delta(*)$ is a semistar operation on $D$, and is called the descent of $*$ to $D$.

Theorem 1. Let $D$ be an integrally closed local ring with dimension $n<\infty$. The following conditions are equivalent.

(1) $D$ is a valuation ring.

(2) $n+1 \leq\left|\Sigma^{\prime}(D)\right| \leq 2 n+1$.

Proof. $\quad(1) \Longrightarrow(2)$ : We rely on the dimension of $D$, and suppose that the assertion holds for each integer $k<n$.

Let $M \supsetneqq P_{n-1} \supsetneqq \cdots \supsetneqq P_{1} \supsetneqq\{0\}$ be the prime ideals of $V=D$. Let $\mathrm{d}_{i}^{\prime}$ be the $\mathrm{d}^{\prime}$-operation on $U_{i}=V_{P_{i}}$, and let $*_{i}$ be the descent of $\mathrm{d}_{i}^{\prime}$ to $V$. Then $\Sigma^{\prime}(V)$ contains $\left\{\mathrm{d}^{\prime}, *_{1}, \cdots, *_{n-1}, \mathrm{e}\right\}$.

Let $\left|\Sigma^{\prime}\left(U_{n-1}\right)\right|=k$ and let $\Sigma^{\prime}\left(U_{n-1}\right)=\left\{*_{1}^{\prime}, \cdots, *_{k}^{\prime}\right\}$, where $k \leq 2(n-1)+1$. Let $*$ be a semistar operation on $V$ with $* \notin\left\{\delta\left(*_{1}^{\prime}\right), \cdots, \delta\left(*_{k}^{\prime}\right)\right\}$, where $\delta\left(*_{i}^{\prime}\right)$ is the descent of $*_{i}^{\prime}$ to $V$. Then $V^{*}=V$. The restriction $\left.*\right|_{F(V)}$ is a star operation on $V$. Proposition 2 implies that either $*=\mathrm{d}^{\prime}$ or $*=\mathrm{v}^{\prime}$. Hence $\left|\Sigma^{\prime}(V)\right| \leq k+2 \leq 2 n+1$.

$(2) \Longrightarrow(1): D$ is the intersection of a family of valuation overrings of $D$. Since $\left|\Sigma^{\prime}(D)\right|<\infty, D$ is the intersection of a finite family of valuation overrings of $D$. Since $D$ is local, $D$ is a valuation ring.

The following is a semigroup version of Theorem 1 .

Theorem 2. Let $S$ be an integrally closed semigroup with dimension $n<\infty$. The following conditions are equivalent.

(1) $S$ is a valuation semigroup.

(2) $n+1 \leq\left|\Sigma^{\prime}(S)\right| \leq 2 n+1$.

We have the following,

Theorem 3. Let $D$ be an integrally closed local ring. The following conditions are equivalent.

(1) $\left|\Sigma^{\prime}(D)\right|<\infty$.

(2) $D$ is a valuation ring with dimension $<\infty$.

The following is a semigroup version of Theorem 3.

Theorem 4. Assume that $S$ is integrally closed. The following conditions are equivalent.

(1) $\left|\Sigma^{\prime}(S)\right|<\infty$.

(2) $S$ is a valuation semigroup with dimension $<\infty$.

Lemma 3. Let $V$ be a valuation semigroup, and let $\Gamma$ be its value group. Let $P$ be a prime ideal of $V$, and let $H$ be the convex subgroup of $\Gamma$ associated to $P$.

(1) The ordered additive group $\Gamma / H$ belongs to the valuation semigroup $V_{P}$. 
(2) The ordered additive group $H$ belongs to the valuation semigroup $V-P$.

Proof. Let $v$ be a valuation on $G=\mathrm{q}(V)$ onto $\Gamma$ belonging to $V$.

(1) Let $w$ be the composition of the mappings $v$ and the canonical mapping $\Gamma \longrightarrow \Gamma / H$. Then $w$ is a valuation on $G$ with value group $\Gamma / H$. Let $w(\alpha) \geq 0$ for $\alpha \in G$. There exists an element $a \in V$ such that $v(\alpha)-v(a) \in H$. There exists an element $s \in V-P$ such that either $v(\alpha)-v(a)=v(s)$ or $v(\alpha)-v(a)=-v(s)$. Hence we have $\alpha \in V_{P}$. Therefore the valuation semigroup of $w$ is $V_{P}$.

(2) The restriction $\left.v\right|_{V-P}$ from $V-P$ to $H$ determines a valuation $w$ on $\mathrm{q}(V-P)$. The valuation semigroup belonging to $w$ is $V-P$.

Theorem 5. Assume that $D$ has dimension $n<\infty$. The following conditions are equivalent.

(1) $D$ is a discrete valuation ring.

(2) $\left|\Sigma^{\prime}(D)\right|=n+1$.

Proof. (1) $\Longrightarrow(2)$ : Let $M$ be the maximal ideal of $V=D$, and let $M \supsetneqq P_{n-1} \supsetneqq$ $\cdots P_{1} \supsetneqq(0)$ be the prime ideals of $V$. Then $U_{i}=V_{P_{i}}$ is a discrete valuation ring for each $i$. Let $*_{i}=*_{U_{i}}$.

We will show that $\Sigma^{\prime}(V)=\left\{\mathrm{e}, *_{1}, \cdots, *_{n-1}, \mathrm{~d}^{\prime}\right\}$ by the induction on $\operatorname{dim}(V)$. Suppose that the assertion holds for each $i<n$.

Let $*$ be a semistar operation on $V$.

The case where $V^{*}=K$, where $K=\mathrm{q}(V)$ : Then we have $*=\mathrm{e}$.

The case where $V^{*}=U_{i}$ for some $i$ : Let $\alpha(*)$ be the ascent of $*$ to $U_{i}$. Then we have $\alpha(*)=\mathrm{d}_{U_{i}}^{\prime}$ by the induction hypothesis. For each $I \in \mathrm{F}^{\prime}(V)$, we have

$I^{*}=(I V)^{*}=\left(I U_{i}\right)^{\alpha(*)}=I U_{i}=I^{*_{i}}$.

That is, $*=*_{i}$.

The case where $V^{*}=V$ : Since $V$ is discrete, $M$ is principal. The restriction $\left.*\right|_{F(V)}$ coincides with d by Proposition 2 . Hence $*=\mathrm{d}^{\prime}$.

Therefore $\left|\Sigma^{\prime}(V)\right|=n+1$.

$(2) \Longrightarrow(1)$ : Let $N$ be a maximal ideal of $\bar{D}$ with height $n$. We have $D=(\bar{D})_{N}$ by the assumption. That is, $D$ is an integrally closed local ring. Then Theorem 3 implies that $V=D$ is a valuation ring. Let $M \supsetneqq P_{n-1} \supsetneqq \cdots \supsetneqq P_{1} \supsetneqq(0)$ be the prime ideals of $V$, and let $U_{i}=V_{P_{i}}$ for each $i$. Let $*_{i}=*_{U_{i}}$ for each $i$.

We will show that $V$ is a discrete valuation ring by the induction on $\operatorname{dim}(V)$. Suppose that the assertion holds for each $i<n$.

Let $\Gamma$ be a value group belonging to $V$, and let $H_{i}$ be the convex subgroup of $\Gamma$ associated to $P_{i}$ for each $i$.

The case where $M$ is principal: Then we have $\left|\Sigma^{\prime}\left(U_{n-1}\right)\right|=n, \Gamma$ has a minimal positive element, and $H_{n-1}$ is order-isomorphic with $\mathbf{Z}$. Since $U_{n-1}$ is discrete, $\Gamma$ is discrete. That is, $V$ is discrete.

The case where $M$ is not principal: Then $\mathrm{v}^{\prime}$ differs from each of $\mathrm{e}, *_{1}, \cdots, *_{n-1}$, $\mathrm{d}^{\prime}$. Hence we have $\left|\Sigma^{\prime}(V)\right|>n+1$; a contradiction.

The following is a semigroup version of Theorem 5 .

Theorem 6. Assume that $S$ has dimension $n<\infty$. The following conditions are equivalent. 
(1) $S$ is a discrete valuation semigroup.

(2) $\left|\Sigma^{\prime}(S)\right|=n+1$.

Theorem 7. Let $V$ be a valuation semigroup with dimension $n<\infty$, let $v$ be its valuation, and let $\Gamma$ be the value group of $v$. Let $M=P_{n} \supsetneqq P_{n-1} \supsetneqq \cdots \supsetneqq P_{1}$ be the prime ideals of $V$, let $H_{n}=\{0\} \varsubsetneqq H_{n-1} \varsubsetneqq \cdots \varsubsetneqq H_{1} \varsubsetneqq \Gamma$ be the convex subgroups of $\Gamma$, and let $U_{i}=V_{P_{i}}$ for each $i$. Let $m$ be a positive integer with $n+1 \leq m \leq 2 n+1$. Then the following conditions are equivalent.

(1) $\left|\Sigma^{\prime}(V)\right|=m$.

(2) The maximal ideal of $U_{i}$ is principal for exactly $2 n+1-m$ of $i$ in $\{1, \cdots, n\}$.

(3) The ordered additive group $\Gamma / H_{i}$ has a minimal positive element for exactly $2 n+1-m$ of $i$ in $\{1, \cdots, n\}$.

Proof. $(2) \Longrightarrow(1)$ : We rely on the induction on $\operatorname{dim}(V)$. Suppose that the assertion holds for each positive integer $<n$.

(i) The case where $M$ is not principal: Since the assertion holds for $U_{n-1}$, and since $2 n+1-m=2(n-1)+1-(m-2)$, we have $\left|\Sigma^{\prime}\left(U_{n-1}\right)\right|=m-2$. Let $\Sigma^{\prime}\left(U_{n-1}\right)=\left\{*_{1}, \cdots, *_{m-2}\right\}$. Then $\delta\left(*_{1}\right), \cdots, \delta\left(*_{m-2}\right), \mathrm{d}^{\prime}$, and $\mathrm{v}^{\prime}$ are distinct with each other, where $\delta\left(*_{i}\right)$ is the descent of $*_{i}$ to $V$. Let $*$ be a semistar operation on $V$.

If $V^{*}=V$, then either $*=\mathrm{d}^{\prime}$ or $*=\mathrm{v}^{\prime}$.

If $\mathrm{q}(V) \supsetneqq V^{*} \supsetneqq V$, we have $V^{*}=V_{P_{i}}$ for some $i<n$. Let $\alpha(*)$ be the ascent of $*$ to $V_{P_{i}}$. Then $*$ is the descent of $\alpha(*)$ to $V$.

It follows that $\left|\Sigma^{\prime}(V)\right|=m$.

(ii) The case where $M$ is principal: Since the assertion holds for $U_{n-1}$, and since $2 n-m=2(n-1)+1-(m-1)$, we have $\left|\Sigma^{\prime}\left(U_{n-1}\right)\right|=m-1$. Let $\Sigma^{\prime}\left(U_{n-1}\right)=\left\{*_{1}, \cdots, *_{m-1}\right\}$. Then $\delta\left(*_{1}\right), \cdots, \delta\left(*_{m-1}\right)$ and $\mathrm{d}^{\prime}$ are distinct with each other. Let $*$ be a semistar operation on $V$.

If $V^{*}=V$, then $*=\mathrm{d}^{\prime}$.

If $\mathrm{q}(V) \supsetneqq V^{*} \supsetneqq V$, we have $V^{*}=V_{P_{i}}$ for some $i<n$. Let $\alpha(*)$ be the ascent of $*$ to $V_{P_{i}}$. Then $*$ is the descent of $\alpha(*)$ to $V$.

Therefore $\left|\Sigma^{\prime}(V)\right|=m$.

$(2) \Longrightarrow(3)$ : Since the value group of $V_{P_{i}}$ is $\Gamma / H_{i}, \Gamma / H_{i}$ has a minimal positive element for exactly $2 n+1-m$ of $i$.

The following is a ring version of Theorem 7 .

Theorem 8. Let $V$ be a valuation domain with dimension $n$, let $v$ be its valuation, and let $\Gamma$ be the value group of $v$. Let $M=P_{n} \supsetneqq P_{n-1} \supsetneqq \cdots \supsetneqq P_{1} \supsetneqq$ (0) be the prime ideals of $V$, and let $H_{n}=\{0\} \varsubsetneqq H_{n-1} \varsubsetneqq \cdots \varsubsetneqq H_{1} \varsubsetneqq \Gamma$ be the convex subgroups of $\Gamma$. Let $m$ be a positive integer with $n+1 \leq m \leq 2 n+1$. Then the following conditions are equivalent.

(1) $\left|\Sigma^{\prime}(V)\right|=m$.

(2) The maximal ideal of $V_{P_{i}}$ is principal for exactly $2 n+1-m$ of $i$ in $\{1, \cdots, n\}$.

(3) $\Gamma / H_{i}$ has a minimal positive element for exactly $2 n+1-m$ of $i$ in $\{1, \cdots, n\}$. 
Example 1. Let $n$ and $m$ be positive integers with $n+1 \leq m \leq 2 n+$ 1. Let $k_{1}, \cdots, k_{2 n+1-m} \in\{1, \cdots, n\}$ be distinct with each other (The set $\left\{k_{1}, \cdots, k_{2 n+1-m}\right\}$ may be empty). Let $G_{i}=\mathbf{Z}$ with the standard order for each $i \in\left\{k_{1}, \cdots, k_{2 n+1-m}\right\}$, and let $G_{j}=\mathbf{R}$ with the standard order for each $j \in\{1, \cdots, n\}-\left\{k_{1}, \cdots, k_{2 n+1-m}\right\}$. Let $\Gamma=G_{1} \oplus \cdots \oplus G_{n}$ be the direct sum of the $G_{i}$, and introduce the lexicographic order on $\Gamma$; Let $x=$ $\left(a_{1}, \cdots, a_{n}\right), y=\left(b_{1}, \cdots, b_{n}\right)$ be elements of $G_{1} \oplus \cdots \oplus G_{n}$ with $a_{n}<b_{n}$, then let $x<y$. There exists a valuation domain $V$ with the value group $\Gamma$. Let $P_{n} \supsetneqq P_{n-1} \supsetneqq \cdots \supsetneqq P_{1} \supsetneqq\{0\}$ be the prime ideals of $V$. Then the maximal ideal of $V_{P_{i}}$ is principal if and only if $G_{n-i+1}$ is discrete. And $G_{n-i+1}$ is discrete if and only if $i \in\left\{n+1-k_{1}, \cdots, n+1-k_{2 n+1-m}\right\}$.

Lemma 4. Let $D$ be a Prüfer ring with exactly two maximal ideals $M$ and $N$. Then $D$ is a Bezout ring.

Proof. Let $v$ (resp. $w$ ) be valuations belonging to $V=D_{M}\left(\right.$ resp. $\left.W=D_{N}\right)$. Let $a$ and $b$ be nonzero elements of $D$.

The case (1) where $v(a) \leq v(b)$ and $w(a) \leq w(b)$ : Then $d=\frac{b}{a} \in D$. Hence $(a, b)=(a)$.

The case (2) where $v(a)<v(b)$ and $w(a)>w(b)$ : Then $v(a+b)=v(a), w(a+$ $b) \leq w(a)$. The case (1) implies that $(a+b, a)=(a+b)$. Hence $(a, b)=(a+b)$.

Proposition 3. Let $D$ be an $n$-dimensional Prüfer ring with $n>2$ and with exactly two maximal ideals $M$ and $N$. Assume that there exist prime ideals $P_{1}, P_{2}, \cdots, P_{n-1}$ of $D$ such that $M \cap N \supsetneqq P_{n-1} \supsetneqq \cdots \supsetneqq P_{2} \supsetneqq P_{1} \supsetneqq(0)$, and that there exist elements $\pi_{1}, \pi_{2}, \cdots, \pi_{n-1}, p$ and $q$ of $D$ such that $P_{i} D_{P_{i}}=\pi_{i} D_{P_{i}}$ for each $i, M=(p)$ and $N=(q)$.

Define the fractional ideals $A_{n-1}=\cup_{1}^{\infty}\left(\frac{1}{\pi_{n-1}^{m}}\right), \cdots, A_{2}=\cup_{1}^{\infty}\left(\frac{1}{\pi_{2}^{m}}\right), A=$ $\cup_{1}^{\infty}\left(\frac{1}{p^{m}}\right), B=\cup_{1}^{\infty}\left(\frac{1}{q^{m}}\right)$ and $C=\cup_{1}^{\infty}\left(\frac{1}{p^{m} q^{m}}\right)$ of $D$.

For each nonzero fractionl ideal $I$ of $D$, let $I^{*_{1}}=I$ if $I$ is of the form $x A$, and let $I^{*_{1}}=I^{v}$ otherwise, where $x \in K=\mathrm{q}(D)$. Let $I^{*_{2}}=I$ if $I$ is of the form $x B$, and let $I^{*_{2}}=I^{v}$ otherwise.

(1) Each element $x$ of $K-\{0\}$ can be expressed as $\pi_{1}^{a_{1}} \pi_{2}^{a_{2}} \cdots \pi_{n-1}^{a_{n-1}} p^{b} q^{c} u$ with integers $a_{i}, b, c$ and $a$ unit $u$ of $D$. This expression is unique for the element $x$.

(2) Each non-pincipal fractional ideal $I$ of $D$ is of the form $x A_{2}$ or $\cdots$ or $x A_{n-1}$ or $x A$ or $x B$ or $x C$ with $x \in K-\{0\}$.

(3) We have $P_{1}=P_{1}^{v}=\pi_{1} A_{2}, P_{2}=P_{2}^{v}=\pi_{2} A_{3}, \cdots$, and $P_{n-2}=P_{n-2}^{v}=$ $\pi_{n-2} A_{n-1}$.

(4) We have $P_{n-1}=P_{n-1}^{v}=\pi_{n-1} C=\pi_{n-1} A^{v}=\pi_{n-1} B^{v}, A \neq A^{v}$, $B \neq B^{v}$, and $A \neq x B$ for each $x \in K$.

(5) $*_{1}$ and $*_{2}$ are star operations on $D$.

(6) $*_{1}, *_{2}, \mathrm{~d}$ and $\mathrm{v}$ are distinct with each other.

Proof. Let $V=D_{M}, W=D_{N}, U_{i}=D_{P_{i}}$ for each $i$, let $M_{i}=P_{i} U_{i}$, let $v$ (resp. $w, u_{i}$ ) be a valuation belonging to $V\left(\right.$ resp. $\left.W, U_{i}\right)$ with $v(p)=1$ (resp. 
$\left.w(q)=1, u_{i}\left(\pi_{i}\right)=1\right)$.

(1) Let $y \in D-\{0\}$. Since $u_{1}\left(M_{1}\right)$ has a minimal element $u_{1}\left(\pi_{1}\right)$, we have $y=\pi_{1}^{k} u$ with $k \geq 0$ and unit $u$ of $U_{1}$.

Let $y \in D-P_{1}$. Since $u_{2}\left(M_{2}\right)$ has a minimal element $u_{2}\left(\pi_{2}\right)$, we have $y=\pi_{2}^{k} u$ with $k \geq 0$ and unit $u$ of $U_{2}$.

Let $y \in D-P_{n-2}$. Since $u_{n-1}\left(M_{n-1}\right)$ has a minimal element $u_{n-1}\left(\pi_{n-1}\right)$, we have $y=\pi_{n-1}^{k} u$ with $k \geq 0$ and unit $u$ of $U_{n-1}$.

Let $y \in D-P_{n-1}$. Since $v(M V)$ (resp. $v(N W)$ ) has a minimal element $v(p)$ (resp. $w(q)$ ), we have $y=p^{k_{1}} q^{k_{2}} u$ with $k_{1} \geq 0, k_{2} \geq 0$ and unit $u$ of $D$.

(2) We may assume that there exist principal fractional ideals $I_{n}=\left(x_{n}\right)$ of $D$ such that $I_{1} \varsubsetneqq I_{2} \varsubsetneqq I_{3} \varsubsetneqq \cdots$ and that $I=\cup_{1}^{\infty} I_{n}$. Each $x_{i}$ is of the form $\pi_{1}^{a_{i, 1}} \pi_{2}^{a_{i, 2}} \cdots \pi_{n-1}^{a_{i, n-1}} p^{b_{i}} q^{c_{i}} u_{i}$ with integers $a_{i, j}, b_{i}, c_{i}$ and unit $u_{i}$ of $D$.

If $\inf _{i} a_{i, 1}=-\infty$, then $I=K$. Hence we may assume that $x_{i}=$ $\pi_{2}^{a_{i, 2}} \cdots \pi_{n-1}^{a_{i, n-1}} p^{b_{i}} q^{c_{i}}$. If inf ${ }_{i} a_{i, 2}=-\infty$, then $I=A_{2}$. If inf ${ }_{i} a_{i, 2}>-\infty$, then we may assume that $x_{i}=\pi_{3}^{a_{i, 3}} \cdots \pi_{n-1}^{a_{i, n-1}} p^{b_{i}} q^{c_{i}}$. If inf ${ }_{i} a_{i, 3}=-\infty$, then $I=A_{3}$. If $\inf _{i} a_{i, 3}>-\infty$, we may assume that $x_{i}=\pi_{4}^{a_{i, 4}} \cdots \pi_{n-1}^{a_{i, n-1}} p^{b_{i}} q^{c_{i}} \cdots$. We may assume that $x_{i}=\pi_{n-1}^{a_{i, n-1}} p^{b_{i}} q^{c_{i}}$. If inf ${ }_{i} a_{i, n-1}=-\infty$, then $I=A_{n-1}$. If inf ${ }_{i} a_{i, n-1}>-\infty$, then we may assume that $x_{i}=p^{b_{i}} q^{c_{i}}$. If $\inf _{i} b_{i}>-\infty$, we may assume that $x_{i}=q^{c_{i}}$. Then $I=B$. If inf ${ }_{i} c_{i}>-\infty$, we may assume that $x_{i}=p^{b_{i}}$. Then $I=A$. If $\inf _{i} b_{i}=\inf _{i} c_{i}=-\infty$, then $I=C$.

(3) We have $P_{1}=\cap_{1}^{\infty}\left(\pi_{2}^{m}\right)$, and hence $P_{1}=P_{1}^{v} ; P_{2}=\cap_{1}^{\infty}\left(\pi_{3}^{m}\right)$, and hence $P_{2}=P_{2}^{v} ; \cdots ; P_{n-2}=\cap_{1}^{\infty}\left(\pi_{n-1}^{m}\right)$, and hence $P_{n-2}=P_{n-2}^{v}$.

Next, $\pi_{n-2} A_{n-1}=\cup_{1}^{\infty}\left(\frac{\pi_{n-2}}{\pi_{n-1}^{m}}\right)=P_{n-2} ; \cdots ; \pi_{1} A_{2}=\cup_{1}^{\infty}\left(\frac{\pi_{1}}{\pi_{2}^{m}}\right)=P_{1}$.

(4) $\quad \pi_{n-1} C=\cup_{1}^{\infty}\left(\frac{\pi_{n-1}}{p^{m} q^{m}}\right)=P_{n-1} . P_{n-1}=\cap_{1}^{\infty}\left(p^{m} q^{m}\right)$, and hence $P_{n-1}=$ $P_{n-1}^{v}$. Assume that $\pi_{n-1} A \subset(\alpha)$ for an element $\alpha \in K$. It follows that $P_{n-1} \subset$ $(\alpha)$. Hence $\pi_{n-1} A^{v}=P_{n-1}$. Similarly, $\pi_{n-1} B^{v}=P_{n-1}$.

Suppose that $A=x B$ for some $x \in K$. Then we have $x=p^{b} q^{c} u$, where $b, c$ are integers and $u$ is a unit of $D$; a contradiction.

(5) Let $I$ and $J$ be nonzero fractional ideals of $D$ such that $I \subset J$. We will show that $I^{*_{1}} \subset J^{*_{1}}$. We may assume that $I \neq x A$ for each $x \in K$, and that $J=A$.

We must show that $I^{v} \subset A$. We may assume that $I \neq I^{v}$. Then (2) and (3) imply that $I=x B$ for some $x \in K-\{0\} ; x B \subset A$. Since $\frac{x}{q^{m}} \in A$ for each $m>0$, we have $w(x) \geq m$ for each $m>0$. Hence we have $x \in P_{n-1}$. Therefore $I \subset\left(\frac{1}{p}\right)$, and hence $I^{v} \subset\left(\frac{1}{p}\right) \subset A$.

Similarly, $*_{2}$ is a star operation on $D$.

(6) Consider $A$ and $B$.

Remark 1. Let $D$ be a 2-dimensional Prüfer ring with exactly two maximal ideals $M$ and $N$. Assume that there exists a prime ideal $P$ of $D$ such that $M \cap N \supsetneqq P \supsetneqq(0)$, and that there exist elements $\pi, p$ and $q$ of $D$ such that 
$P D_{P}=\pi D_{P}, M=(p)$ and $N=(q)$. Define the fractional ideals $A=\cup_{1}^{\infty}\left(\frac{1}{p^{m}}\right)$, $B=\cup_{1}^{\infty}\left(\frac{1}{q^{m}}\right)$ and $C=\cup_{1}^{\infty}\left(\frac{1}{p^{m} q^{m}}\right)$ of $D$.

For each nonzero fractionl ideal $I$ of $D$, let $I^{*_{1}}=I$ if $I$ is of the form $x A$, and let $I^{*_{1}}=I^{v}$ otherwise, where $x \in K=\mathrm{q}(D)$. Let $I^{*_{2}}=I$ if $I$ is of the form $x B$, and let $I^{*_{2}}=I^{v}$ otherwise.

(1) Each element $x$ of $K-\{0\}$ can be expressed as $\pi^{a} p^{b} q^{c} u$ with integers $a, b, c$ and unit $u$ of $D$. This expression is unique for the element $x$.

(2) Each non-principal fractional ideal $I$ of $D$ is of the form $x A$ or $x B$ or $x C$ with $x \in K-\{0\}$.

(3) We have $P=P^{v}=\pi C=\pi A^{v}=\pi B^{v}, A \neq A^{v}$, and $B \neq B^{v}$.

(4) $A \neq x B$ for each $x \in K$.

(5) $\quad *_{1}$ and $*_{2}$ are star operations on $D$.

(6) $*_{1}, *_{2}, \mathrm{~d}$ and $\mathrm{v}$ are distinct with each other.

Theorem 9. Let $D$ be an $n$-dimensional Prüfer ring with $n>1$ and with exactly two maximal ideals $M$ and $N$. Assume that there exist prime ideals $P_{1}, P_{2}, \cdots, P_{n-1}$ of $D$ such that $M \cap N \supsetneqq P_{n-1} \supsetneqq \cdots \supsetneqq P_{1} \supsetneqq(0)$, and that $P_{i} D_{P_{i}}$ is a principal ideal of $U_{i}=D_{P_{i}}$ for each $i, M$ and $N$ are principal ideals of $D$.

Then we have $\left|\Sigma^{\prime}(D)\right|=n+6$, and $\Sigma^{\prime}(D)=\left\{\mathrm{e}, *_{U_{1}}, \cdots, *_{U_{n-1}}, *_{V}, *_{W}, \mathrm{~d}^{\prime}, \mathrm{v}^{\prime}\right.$, $\left.*_{1}^{\prime}, *_{2}^{\prime}\right\}$, where $*_{1}^{\prime}$ (resp. $\left.*_{2}^{\prime}\right)$ is the canonical extension of $*_{1}$ (resp. $*_{2}$ ) in Proposition 3 or in Remark 1 to a semistar operation on $D$, and $V=D_{M}, W=D_{N}$.

Proof. $\quad$ Suppose that there exists a semistar operation $*^{\prime}$ on $D$ such that $*^{\prime} \notin$ $\left\{\mathrm{e}, *_{U_{1}}, \cdots, *_{U_{n-1}}, *_{V}, *_{W}, \mathrm{~d}^{\prime}, \mathrm{v}^{\prime}, *_{1}^{\prime}, *_{2}^{\prime}\right\}$.

If $D^{*^{\prime}}=K=\mathrm{q}(D)$, then $*^{\prime}=\mathrm{e}$.

If $D \varsubsetneqq D^{*^{\prime}} \varsubsetneqq K$, then $R=D^{*^{\prime}}$ is a member in $\left\{U_{1}, \cdots, U_{n-1}, V, W\right\}$. We have $\alpha_{R / D}\left(*^{\prime}\right)=\mathrm{d}_{R}^{\prime}$, and hence $*^{\prime}=*_{R}$.

Hence we may suppose that $D^{*^{\prime}}=D$. Then $\left.*^{\prime}\right|_{F(D)}=*$ is a star operation on $D$. Then $* \notin\left\{\mathrm{d}, \mathrm{v}, *_{1}, *_{2}\right\}$ by the assumption.

The case where $A^{*}=A, B^{*}=B$ in Proposition 3 or Remark 1: Then $*=\mathrm{d}$; a contradiction.

The case where $A^{*}=A, B^{*}=B^{v}$ : Then $*=*_{1}$; a contradiction.

The case where $A^{*}=A^{v}, B^{*}=B$ : Then $*=*_{2}$; a contradiction.

The case where $A^{*}=A^{v}, B^{*}=B^{v}$ : Then $*=\mathrm{v} ;$ a contradiction.

The case where $A \varsubsetneqq A^{*} \varsubsetneqq A^{v}$ : Note that $A^{v}=\cup\left(\frac{1}{p^{m} q^{m}}\right)$.

If $A^{*} \subset\left(\frac{1}{p^{m} q^{m}}\right)$ for some $m$, then $A^{v} \subset\left(\frac{1}{p^{m} q^{m}}\right)$; a contradiction. Therefore, for each $m$, there exists $n>m$ such that $\left(\frac{1}{p^{n} q^{n}}\right)$ contains an element $x_{m}$ of $A^{*}$ with $x_{m} \notin\left(\frac{1}{p^{n-1} q^{n-1}}\right)$. Then $x_{m}=\frac{u}{p^{k} q^{k^{\prime}}}$, where $k, k^{\prime} \leq n$ and $u$ is a unit of $D$. 
If $k, k^{\prime} \leq n-1$, then $x_{m} \in\left(\frac{1}{p^{n-1} q^{n-1}}\right)$; a contradiction. Hence either $k$ or $k^{\prime}$, say $k^{\prime}$, equals to $n ; x_{m}=\frac{u}{p^{k} q^{n}}$ for some $k \leq n$. Then we have

$$
\frac{1}{p^{n} q^{n}} \in \frac{1}{p^{n} q^{n}} D=\frac{1}{p^{n} q^{n}}\left(p^{n-k}, q^{n}\right)=\left(\frac{1}{p^{k} q^{n}}, \frac{1}{p^{n}}\right) \subset A^{*}
$$

and hence we have $y_{m}=\frac{1}{p^{n} q^{n}} \in A^{*}$. Then we have $\cup_{m}\left(\frac{1}{p^{m} q^{m}}\right) \subset \cup_{m}\left(y_{m}\right) \subset A^{*}$, and hence $A^{v} \subset A^{*}$; a contradiction.

Proposition 4. Let $D$ be an $n$-dimensional Prüfer ring with $n>1$ and with exactly two maximal ideals $M$ and $N$. Assume that there exist prime ideals $P_{1}, P_{2}, \cdots, P_{n-1}$ of $D$ such that $M \cap N \supsetneqq P_{n-1} \supsetneqq \cdots \supsetneqq P_{2} \supsetneqq P_{1} \supsetneqq(0)$, and that there exist elements $p$ and $q$ of $D$ such that $M=(p)$ and $N=(q)$.

Define the fractional ideals $A=\cup_{1}^{\infty}\left(\frac{1}{p^{m}}\right), B=\cup_{1}^{\infty}\left(\frac{1}{q^{m}}\right)$, and $C=\cup_{1}^{\infty}$ $\left(\frac{1}{p^{m} q^{m}}\right)$.

For each nonzero fractional ideal $I$ of $D$, let $I^{*_{1}}=I$ if $I$ is of the form $x A$, and let $I^{*_{1}}=I^{v}$ otherwise, where $x \in K=\mathrm{q}(D)$. Let $I^{*_{2}}=I$ if $I$ is of the form $x B$, and let $I^{*_{2}}=I^{v}$ otherwise.

(1) $P_{1}=P_{1}^{v}, \cdots, P_{n-1}=P_{n-1}^{v}$.

(2) $C=A^{v}=B^{v}, A \neq A^{v}$, and $B \neq B^{v}$. $A \neq x B$ for each $x \in K$.

(3) $*_{1}$ and $*_{2}$ are star operations on $D$.

(4) $*_{1}, *_{2}, \mathrm{~d}$ and $\mathrm{v}$ are distinct with each other.

Proof. (1) We have $P_{1}=\cap\left\{(\pi) \mid \pi \in P_{2}-P_{1}\right\}$, hence $P_{1}=P_{1}^{v} ; \cdots ; P_{n-2}=$ $\cap\left\{(\pi) \mid \pi \in P_{n-1}-P_{n-2}\right\}$, hence $P_{n-2}=P_{n-2}^{v}$.

We have $P_{n-1}=\cap\left(p^{m} q^{m}\right)$, hence $P_{n-1}=P_{n-1}^{v}$.

(2) Let $x \in\left(\frac{p^{m} q^{m}}{\pi}\right)$ for $\pi \in P_{n-1}-P_{n-2}$ and $m \geq 0$. Then $v\left(x^{-1}\right) \leq v(\pi)-$ $m v(p)$. It follows that $v\left(x^{-1}\right) \leq v\left(p^{n}\right)$ for some $n$. Similarly, $w\left(x^{-1}\right) \leq w\left(q^{n^{\prime}}\right)$ for some $n^{\prime}$. Therefore $x \in C$. It follows that $C=\cap\left\{\left(\frac{p^{m} q^{m}}{\pi}\right) \mid \pi \in P_{n-1}-P_{n-2}\right.$ and $m \geq 0\}$.

(3) Let $I$ be a nonzero fractional ideal of $D$ with $I \subset A$ such that $I \neq x A$ for each $x \in K$. We must show that $I^{v} \subset A$. We may assume that $I \not \subset\left(\frac{1}{p^{m}}\right)$ for each $m>0$. Choose $x_{m} \in I-\left(\frac{1}{p^{m}}\right)$. Then $x_{m} \in\left(\frac{1}{p^{n}}\right)-\left(\frac{1}{p^{n-1}}\right)$ for some $n>m$. Then $x_{m}=\frac{q^{k} u}{p^{n}}$ for $k \geq 0$ and unit $u$ of $D$. Let $k_{0}=\min \left\{k \geq 0 \mid q^{k} \in I\right\}$. It follows that $I=q^{k_{0}} A$; a contradiction.

Proposition 5. Let $D$ be an $n$-dimensional Prüfer ring with $n>2$ and with exactly two maximal ideals $M$ and $N$. Assume that there exist prime ideals $P_{1}, P_{2}, \cdots, P_{n-1}$ of $D$ such that $M \cap N \supsetneqq P_{n-1} \supsetneqq \cdots \supsetneqq P_{2} \supsetneqq P_{1} \supsetneqq(0)$, and 
that there exist elements $\pi_{1}, \pi_{2}, \cdots, \pi_{n-1}$ and $p$ of $D$ such that $P_{i} D_{P_{i}}=\pi_{i} D_{P_{i}}$ for each $i, M=(p)$, and that $N$ is not a principal ideal.

Let $A_{n-1}=\cup_{1}^{\infty}\left(\frac{1}{\pi_{n-1}^{m}}\right), \cdots, A_{2}=\cup_{1}^{\infty}\left(\frac{1}{\pi_{2}^{m}}\right), A=\cup_{1}^{\infty}\left(\frac{1}{p^{m}}\right), B=\cup\left\{\left(\frac{1}{q}\right) \mid q \in\right.$ $N-M\}$ and $C=\cup\left\{\left(\frac{1}{p^{m} q}\right) \mid q \in N-M\right.$ and $\left.m \geq 0\right\}$.

For each nonzero fractional ideal $I$ of $D$, let $I^{*}=I$ if $I=x B$ for some $x \in K=\mathrm{q}(D)$, and let $I^{*}=I^{v}$ otherwise.

(1) Each element $x$ of $K-\{0\}$ can be expressed as $\pi_{1}^{a_{1}} \pi_{2}^{a_{2}} \cdots \pi_{n-1}^{a_{n-1}} p^{b} u$ with integers $a_{i}, b$ and with unit $u$ of $D_{M}$. This expression is unique for the element $x$.

(2) We have $P_{1}=P_{1}^{v}=\pi_{1} A_{2}, P_{2}=P_{2}^{v}=\pi_{2} A_{3}, \cdots$, and $P_{n-2}=P_{n-2}^{v}=$ $\pi_{n-2} A_{n-1}$.

(3) $\quad P_{n-1}=P_{n-1}^{v}=\pi_{n-1} C=\pi_{n-1} A^{v}=\pi_{n-1} B^{v}, A \neq A^{v}$, and $B \neq B^{v}$. $A \neq x B$ for each $x \in K$.

(4) * is a star operation on $D$.

(5) *, d and $\mathrm{v}$ are distinct with each other.

Proof. Let $V=D_{M}, W=D_{M}$, and $U_{i}=D_{P_{i}}$ for each $i$, let $M_{i}=P_{i} U_{i}$, and let $v$ (resp. $\left.w, u_{i}\right)$ be the valuation belonging to $V$ (resp. $\left.W, U_{i}\right)$. Let $v(p)=1$ and $u_{i}\left(\pi_{i}\right)=1$ for each $i$, and let $\Gamma^{\prime}$ be the value group of $w$.

(2) We have $P_{n-1}=\cap\left\{\left(p^{m} q\right) \mid q \in N-M\right.$ and $\left.m \geq 0\right\}$.

(4) Assume that $I \subset J$ for $I, J \in \mathrm{F}(D)$. It sufficies to show that $I^{*} \subset J^{*}$.

We may assume that $J=B$ and $I \neq x B$ for each $x \in K$.

Let $S=\left\{x_{\lambda} \mid \Lambda\right\}$ be a generating system for $I$. Let $x_{\lambda}=\pi_{1}^{a_{\lambda, 1}} \cdots \pi_{n-1}^{a_{\lambda, n-1}} p^{b_{\lambda}} u_{\lambda}$, where $a_{\lambda, i}, b_{\lambda}$ are integers and $u_{\lambda}$ is a unit of $V$.

If $\inf _{\lambda} a_{\lambda, 1}=-\infty$, then $I=K$; a contradiction. Therefore we may assume that $a_{\lambda, 1}=a_{1}$ does not depend on $\lambda$.

If inf ${ }_{\lambda} a_{\lambda, 2}=-\infty$, then $I \frac{1}{\pi_{1}^{a_{1}}}=A_{2}$. It follows that $I^{v}=I \subset B$. Therefore we may assume that $a_{\lambda, 2}=a_{2}$ does not depend on $\lambda$.

If inf ${ }_{\lambda} a_{\lambda, n-1}=-\infty$, then $I \frac{1}{\pi_{1}^{a_{1}} \cdots \pi_{n-2}^{a_{n-2}}}=A_{n-1}$. It follows that $I^{v}=I \subset B$. Therefore we may assume that $a_{\lambda, n-1}=a_{n-1}$ does not depend on $\lambda$.

We have $x_{\lambda}=\pi_{1}^{a_{1}} \cdots \pi_{n-1}^{a_{n-1}} p^{b_{\lambda}} u_{\lambda}$.

Assume that $a_{1}=\cdots=a_{m-1}=0 \neq a_{m}$ for some $m$. If $a_{m}<0$, then $x_{\lambda} \notin B$; a contradiction. If $a_{m}>0$, then $I \subset D$. Hence $I^{v} \subset B$.

Assume that $a_{1}=\cdots=a_{n-1}=0$. We have $b_{\lambda}=k_{\lambda} \geq 0$ and $x_{\lambda}=p^{k_{\lambda}} u_{\lambda}$.

Assume that inf ${ }_{\lambda}^{\Gamma^{\prime}} w\left(u_{\lambda}\right)>-\infty$. There exists $q_{0} \in N-M$, such that $w\left(u_{\lambda}\right)>-w\left(q_{0}\right)$ for each $\lambda$. Then $x_{\lambda} \in\left(\frac{1}{q_{0}}\right)$, and hence $I \subset\left(\frac{1}{q_{0}}\right)$. It follows that $I^{v} \subset B$.

Assume that inf ${ }_{\lambda}^{\Gamma^{\prime}} w\left(u_{\lambda}\right)=-\infty$. Since $D$ is a Bezout ring, we may assume that $x_{\lambda}=\frac{p^{k_{\lambda}}}{q_{\lambda}}$ for each $\lambda$. We have $\sup { }_{\lambda}^{\Gamma^{\prime}} w\left(q_{\lambda}\right)=\infty$. Let $k_{0}=\min { }_{\lambda}\{k \geq$ 
$\left.0 \mid p^{k} \in I\right\}$. Since $\frac{p^{k_{\lambda}-k_{0}}}{q_{\lambda}} \in B$, we have $x_{\lambda} \in p^{k_{0}} B$. Hence $I \subset p^{k_{0}} B$.

Let $q_{1} \in N-M$. Choose $\lambda$ such that $w\left(q_{\lambda}\right)>w\left(q_{1}\right)$. We have

$\frac{p^{k_{0}}}{q_{1}} \in\left(\frac{p^{k_{0}}}{q_{\lambda}}\right)=\frac{p^{k_{0}}}{q_{\lambda}}\left(q_{\lambda}, p^{k_{\lambda}-k_{0}}\right)=\left(p^{k_{0}}, \frac{p^{k_{\lambda}}}{q_{\lambda}}\right) \subset I$. Therefore $p^{k_{0}} B \subset I$. It follows that $I=p^{k_{0}} B$; a contradiction.

Remark 2. Let $D$ be a 2-dimensional Prüfer ring with exactly two maximal ideals $M$ and $N$. Assume that there exists a prime ideal $P$ such that $M \cap N \supsetneqq$ $P \supsetneqq(0)$, and that there exists elements $\pi$ and $p$ of $D$ such that $P D_{P}=\pi D_{P}$ and $M=(p)$, and that $N$ is not a principal ideal.

Let $A=\cup_{1}^{\infty}\left(\frac{1}{p^{m}}\right), B=\cup\left\{\left(\frac{1}{q}\right) \mid q \in N-M\right\}$ and $C=\cup\left\{\left(\frac{1}{p^{m} q}\right) \mid q \in N-M\right.$ and $m \geq 0\}$.

For each nonzero fractional ideal $I$ of $D$, let $I^{*}=I$ if $I=x B$ for some $x \in K=\mathrm{q}(D)$, and let $I^{*}=I^{v}$ otherwise.

(1) Each element $x$ of $K-\{0\}$ can be expressed as $\pi^{a} p^{b} u$ with integers $a, b$ and with unit $u$ of $D_{M}$. This expression is unique for the element $x$.

(2) We have $P=P^{v}=\pi C=\pi A^{v}=\pi B^{v}, A \neq A^{v}$, and $B \neq B^{v}$.

(3) $\quad A \neq x B$ for each $x \in K$.

(4) * is a star operation on $D$.

(5) $*, \mathrm{~d}$ and $\mathrm{v}$ are distinct with each other.

Proposition 6. Let $D$ be a 1-dimensional Prüfer ring with exactly two maximal ideals $M$ and $N$.

(1) If either $M$ or $N$, say $N$, is not principal, then $\left|\Sigma^{\prime}(D)\right| \geq 6$.

(2) Assume that $M=(p)$ and $N=(q)$ are principal ideals of $D$. Then $\left|\Sigma^{\prime}(D)\right|=7$.

Proof. Let $v$ (resp. $w$ ) be a valuation on $K=\mathrm{q}(D)$ belonging to $V=D_{M}$ (resp. $W=D_{N}$ ), and let $v(p)=1$.

(1) We have $\left\{\mathrm{e}, *_{V}, *_{W}, \delta^{\prime}\left(\mathrm{v}_{W}^{\prime}\right), \mathrm{d}^{\prime}, \mathrm{v}^{\prime}\right\} \subset \Sigma^{\prime}(D)$.

(2) Let $A=\cup\left(\frac{1}{p^{m}}\right)$ and $B=\cup\left(\frac{1}{q^{m}}\right)$. Let $w(q)=1$, and let $I \in \mathrm{F}^{\prime}(D)$.

(i) The case where $v(I)$ and $w(I)$ are bounded below: Let $a=\min v(I)$ and $b=\min w(I)$. Then $p^{a} q^{b} \in I$. It follows that $I=\left(p^{a} q^{b}\right)$.

(ii) The case where $v(I)$ is bounded below and $w(I)$ is not bounded below: Let $a=\min v(I)$. Then there exists a generating system $\left\{p^{a} q^{b_{\lambda}} \mid \Lambda\right\}$ for $I$. Then $I=p^{a}\left(q^{b_{\lambda}} \mid \lambda\right)=p^{a} B$.

(iii) The case where $v(I)$ is not bounded below and $w(I)$ is bounded below: Let $b=\min w(I)$. Then we have $I=q^{b} A$.

(iv) The case where neither $v(I)$ nor $w(I)$ is bounded below: Then we have $I=K$.

It follows that each fractional ideal of $D$ is principal, and hence $\Sigma(D)=\{\mathrm{d}\}$. Furthermore, $\mathrm{F}^{\prime}(D)-\mathrm{F}(D)=\{x A, x B, K \mid x \in K-\{0\}\}$.

Let $*$ be a semistar operation with $A^{*} \subsetneq K$. We have $A^{*}=x A$ for some $x \in K-\{0\}$. Since $\left(A^{*}\right)^{*}=A^{*}$, we have $A^{*}=A$. Hence $A^{*}$ is either $A$ or $K$. Similarly, $B^{*}$ is either $B$ or $K$. 
Let $A^{*_{1}}=B^{*_{1}}=K$. Then there is determined a semistar operation $*_{1}$ on $D$ extending $\mathrm{d}$.

Let $A^{*_{2}}=A$ and $B^{*_{2}}=K$. Then there is determined a semistar operation $*_{2}$ on $D$ extending d.

Let $A^{*_{3}}=K$ and $B^{*_{3}}=B$. Then there is determined a semistar $*_{3}$ on $D$ extending $\mathrm{d}$.

Let $A^{*_{4}}=A$ and $B^{*_{4}}=B$. Then there is determined a semistar $*_{4}=\mathrm{d}^{\prime}$ on $D$ extending d.

It follows that $\left|\Sigma^{\prime}(D)\right|=7$.

Proposition 7. (1) Let $D$ be a 2-dimensional Prüfer ring with exactly two maximal ideals $M$ and $N$. Then we have $\left|\Sigma^{\prime}(D)\right| \geq 7$.

(2) Let $D$ be a 3-dimensional Prüfer ring with exactly two maximal ideals $M$ and $N$. Then we have $\left|\Sigma^{\prime}(D)\right| \geq 8$.

(3) Let $D$ be a 4-dimensional Prüfer ring with exactly two maximal ideals $M$ and $N$. Then we have $\left|\Sigma^{\prime}(D)\right| \geq 10$.

Proof. (1) Let height $(M)=2$, and let $P$ be a prime ideal of $D$ such that $M \supsetneqq P \supsetneqq(0)$. Let $V=D_{M}, W=D_{N}$ and $U=D_{P}$.

(i) The case where $U, V$ and $W$ have principal maximal ideals and $P \subset N$ : Then we have $\left|\Sigma^{\prime}(D)\right|=8$ by Theorem 9 .

(ii) The case where either $U$ or $V$ or $W$, say $T$, has non-principal maximal ideal and $P \subset N$ : There arises a semistar operaton $\delta\left(\mathrm{v}_{T}^{\prime}\right)$ on $D$. It follows that $|\Sigma(D)| \geq 7$.

(iii) The case where either $U$ or $V$ or $W$, say $T$, has a non-principal maximal ideal and height $(N)=1$ : Let $R=U \cap W$. There arise semistar operations $*_{R}$ and $\delta\left(\mathrm{v}_{T}^{\prime}\right)$.

(iv) The case where $U$ and $W$ have the principal maximal ideals and height $(N)=1$ : Let $R=U \cap W$. We have $\left|\Sigma^{\prime}(R)\right|=7$ by Proposition 6 .

(v) The case where $P \not \subset N$ and height $(N)=2$ : Let $R=U \cap W$. We have $\left|\Sigma^{\prime}(R)\right| \geq 7$ by (iii) and (iv).

(2) Let height $(M)=3$. There exist prime ideals $P_{1}, P_{2}$ such that $M \supsetneqq$ $P_{2} \supsetneqq P_{1} \supsetneqq(0)$. Let $V=D_{M}, U_{2}=D_{P_{2}}, U_{1}=D_{P_{1}}$, and $W=D_{N}$.

(i) The case where height $(N) \leq 2$ : Let $R=U_{2} \cap W$. We have $\left|\Sigma^{\prime}(R)\right| \geq 7$ by (1).

(ii) The case where height $(N)=3$ and $P_{2} \not \subset N$ : Let $Q$ be a prime ideal of $D$ with height 2 such that $Q \subset N$. Let $R=U_{2} \cap D_{Q}$. Then $\left|\Sigma^{\prime}(R)\right| \geq 7$ by (1).

(iii) The case where $U_{1}, U_{2}, V$, and $W$ have principal maximal ideals and $P_{2} \subset N$ : Then $\left|\Sigma^{\prime}(D)\right| \geq 9$ by Theorem 9 .

(iv) The case where either $U_{1}$ or $U_{2}$ or $V$ or $W$, say $T$, has a non-principal maximal ideal and $P_{2} \subset N$ : Then there arises the semistar operation $\delta\left(\mathrm{v}_{T}^{\prime}\right)$ on $D$.

(3) Let height $(M)=4$, and let $P_{1}, P_{2}$, and $P_{3}$ be prime ideals of $D$ with $M \supsetneqq P_{3} \supsetneqq P_{2} \supsetneqq P_{1} \supsetneqq(0)$, and let $V=D_{M}, U_{3}=D_{P_{3}}, U_{2}=D_{P_{2}}, U_{1}=D_{P_{1}}$ and $W=D_{N}$.

(i) The case where height $(N) \leq 3$ : Let $R=U_{3} \cap W$. Then $\left|\Sigma^{\prime}(R)\right| \geq 8$ by (2). 
(ii) The case where height $(N)=4$ and $P_{3} \not \subset N$ : Let $Q$ be a prime ideal of $D$ with height 3 such that $Q \subset N$. Let $R=U_{3} \cap D_{Q}$. Then $\left|\Sigma^{\prime}(R)\right| \geq 8$ by (2).

(iii) The case where neither $M V$ nor $N W$ is principal, and $P_{3} \subset N$ : Then $\Sigma^{\prime}(D)$ contains $\left\{\mathrm{e}, *_{U_{1}}, *_{U_{2}}, *_{U_{3}},{ }^{*}, \delta\left(\mathrm{v}_{V}^{\prime}\right), *_{W}, \delta\left(\mathrm{v}_{W}^{\prime}\right), \mathrm{d}^{\prime}, \mathrm{v}^{\prime}\right\}$, and hence $\left|\Sigma^{\prime}(D)\right| \geq 10$.

(iv) The case where $M V$ is principal, $N W$ is not principal, some member $T$ in $\left\{U_{1}, U_{2}, U_{3}\right\}$ has a non-principal maximal ideal, and $P_{3} \subset N$ : Then $\Sigma^{\prime}(D)$ contains $\left\{\mathrm{e}, *_{U_{1}}, *_{U_{2}}, *_{U_{3}}, \delta\left(\mathrm{v}_{T}^{\prime}\right), *_{V}, *_{W}, \delta\left(\mathrm{v}_{W}^{\prime}\right), \mathrm{d}^{\prime}, \mathrm{v}^{\prime}\right\}$, and hence $\left|\Sigma^{\prime}(D)\right| \geq 10$.

(v) The case where $M V$ is principal, $N W$ is not principal, each member in $\left\{U_{1}, U_{2}, U_{3}\right\}$ has a principal maximal ideal, and $P_{3} \subset N$ : Let $*$ be the star operation on $D$ in Proposition 5 , and let $*^{\prime}$ be an extension of $*$ to a semistar operation on $D$. Then $\Sigma^{\prime}(D)$ contains $\left\{\mathrm{e}, *_{U_{1}}, *_{U_{2}}, *_{U_{3}}, *_{V}, *_{W}, \delta\left(\mathrm{v}_{W}^{\prime}\right), \mathrm{d}^{\prime}, \mathrm{v}^{\prime}, *^{\prime}\right\}$, and hence $\left|\Sigma^{\prime}(D)\right| \geq 10$.

(vi) The case where $M V$ is principal, $N W$ is principal, and $P_{3} \subset N$ : Let $*_{1}, *_{2}$ be the star operations on $D$ in Proposition 4 , and let $*_{1}^{\prime}, *_{2}^{\prime}$ be extensions of $*_{1}^{\prime}, *_{2}^{\prime}$ to semistar operations on $D$. Then $\Sigma^{\prime}(D)$ contains $\left\{\mathrm{e}, *_{U_{1}}, *_{U_{2}}, *_{U_{3}}, *_{V}, *_{W}\right.$, $\left.\mathrm{d}^{\prime}, \mathrm{v}^{\prime}, *_{1}^{\prime}, *_{2}^{\prime}\right\}$, and hence $\left|\Sigma^{\prime}(D)\right| \geq 10$.

Theorem 10. Let $D$ be an integrally closed ring with dim $n \leq 4$. If $n+1 \leq$ $\left|\Sigma^{\prime}(D)\right| \leq 2 n+1$, then $D$ is a valuation ring.

Proof. Suppose the contrary. Then $D$ is not a local ring by Theorem 3 . We may assume that $D$ is a Prüfer ring which has exactly two maximal ideals $M$ and $N$ with height $(M)=n$. Proposition 7 implies a contradiction.

Theorem 11. Let $D$ be $n$-dimensional with $n>0$ which is not local. Then $\left|\Sigma^{\prime}(D)\right| \geq n+5$.

Proof. We rely on the induction on $\operatorname{dim}(D)$. We may assume that the assertion holds for each $i<n$. We may assume that $D$ is a Prüfer ring with exactly two maximal ideals $M$ and $N$ with height $(M)=n$. Let $M \supsetneqq P_{n-1} \supsetneqq \cdots \supsetneqq P_{1} \supsetneqq(0)$ be prime ideals of $D$. Let $V=D_{M}, W=D_{N}, U_{n-1}=D_{P_{n-1}}, \cdots$, and $U_{1}=D_{P_{1}}$.

(1) The case where $P_{n-1} \not \subset N$ and height $(N) \leq n-1$ : Let $R=U_{n-1} \cap W$. Then $\left|\Sigma^{\prime}(R)\right| \geq n+4$.

(2) The case where $P_{n-1} \not \subset N$ and height $(N)=n$ : Let $Q$ be a prime ideal of $D$ with height $n-1$ such that $Q \subset N$. Let $R=U_{n-1} \cap D_{Q}$. We have $\left|\Sigma^{\prime}(R)\right| \geq n+4$.

(3) The case where $P_{n-1} \subset N$, and some member $T$ in $\left\{U_{1}, \cdots, U_{n-1}\right.$, $V, W\}$ has a non-principal maximal ideal: Then $\Sigma^{\prime}(D)$ contains $\left\{\mathrm{e},{ }^{*} U_{1}, \cdots\right.$, $\left.*_{U_{n-1}}, *_{V}, *_{W}, \mathrm{~d}^{\prime}, \mathrm{v}^{\prime}, \delta\left(\mathrm{v}_{T}^{\prime}\right)\right\}$, and hence $\left|\Sigma^{\prime}(D)\right| \geq n+5$.

(4) The case where $P_{n-1} \subset N$, and each member in $\left\{U_{1}, \cdots, U_{n-1}, V, W\right\}$ has a principal maximal ideal: Let $*_{1}, *_{2}$ be the star operations in Proposition 3. Then $\Sigma^{\prime}(D)$ contains $\left\{\mathrm{e}, *_{U_{1}}, \cdots, *_{U_{n-1}}, *_{V}, *_{W}, \mathrm{~d}^{\prime}, \mathrm{v}^{\prime}, *_{1}^{\prime}, *_{2}^{\prime}\right\}$, and hence $\left|\Sigma^{\prime}(D)\right| \geq n+6$.

Theorem 12. For each positive integer $n$ with $n \geq 5$, there exists an integrally closed domain $D$ with dimension $n$ such that $n+1 \leq\left|\Sigma^{\prime}(D)\right| \leq 2 n+1$ and which is not a valuation ring.

Proof. Let $D$ be an integral domain with dimension $n \geq 5$ in Theorem 9 . 
Example 2. Let $n$ be a positive integer, let $A_{1}, A_{2}, B_{1}, \cdots, B_{n-1}$ be nonzero subgroups of $\mathbf{R}$, and introduce on each of them the canonical order. Let $B=$ $B_{1} \oplus \cdots \oplus B_{n-1}$ be the direct sum, and introduce on $B$ the lexicographic order; Let $x=\left(b_{1}, \cdots, b_{n-1}\right), x^{\prime}=\left(b_{1}^{\prime}, \cdots, b_{n-1}^{\prime}\right)$ be elements of $B_{1} \oplus \cdots \oplus B_{n-1}$ with $b_{n-1}<b_{n-1}^{\prime}$, then let $x<x^{\prime}$. Let $v_{0}$ (resp. $w_{0}$ ) be the projection mapping of $A_{1} \oplus A_{2} \oplus B$ to the ordered group $A_{1} \oplus B$ (resp. $A_{2} \oplus B$ ). Let $k$ be a field, and let $K=\mathrm{q}\left(k\left[X ; A_{1} \oplus A_{2} \oplus B\right]\right)$. Let $v$ (resp. $w$ ) be the canonical extension of the valuation $v_{0}$ (resp. $w_{0}$ ) to the valuation on $K$, and let $V$ (resp. $W$ ) be the $n$-dimensional valuation ring on $K$ belonging to $v$ (resp. $w$ ), and let $M^{\prime}$ (resp. $N^{\prime}$ ) be the maximal ideal of $V$ (resp. $W$ ). For each $i$ with $1 \leq i \leq n-1$, let $u_{i}^{\prime}$ be the projection mapping of $A_{1} \oplus A_{2} \oplus B_{1} \oplus \cdots \oplus B_{n-1}$ to the ordered group $B_{n-i} \oplus \cdots \oplus B_{n-1}$, and let $u_{i}$ be the canonical extension of the valuation $u_{i}^{\prime}$ to a valuation on $K$. Let $U_{i}$ be the valuation ring on $K$ belonging to $u_{i}$, and let $M_{i}^{\prime}$ be the maximal ideal of $U_{i}$. Let $D=V \cap W$, let $M=M^{\prime} \cap D, N=N^{\prime} \cap D$, and let $P_{i}=M_{i}^{\prime} \cap D$ for each $i$. Then we have $V=D_{M}, M^{\prime}=M V, W=D_{N}, N^{\prime}=N W$, and $U_{i}=D_{P_{i}}, M_{i}^{\prime}=P_{i} U_{i}$ for each $i$. It follws that $M^{\prime}$ (resp. $N^{\prime}$ ) is a principal ideal of $V$ (resp. $W$ ) if and only if $A_{1}$ (resp. $A_{2}$ ) is a discrete subgroup of $\mathbf{R}$, and that $M_{i}^{\prime}$ is a principal ideal of $U_{i}$ if and only if $B_{n-i}$ is a discrete subgroup of $\mathbf{R}$.

Let $P$ be a prime ideal of $S$. If $a+b \in P$ implies either $a \in P$ or $b \in P$ for elements $a, b \in \mathrm{q}(S)$, then $P$ is called a strongly prime ideal of $S$. If each prime ideal of $S$ is a strongly prime ideal, then $S$ is called a pseudo-valuation semigroup (or a PVS).

Lemma 5. Let $M$ be a maximal ideal of $S$, and let $G=\mathrm{q}(S)$.

(1) If $M$ is strongly prime, then $S$ is a PVS.

(2) If $S$ is a PVS, then there exists a valuation oversemigroup $V$ of $S$ with maximal ideal $M$.

(3) If $S$ is a $P V S$, then $\mathrm{F}^{\prime}(S)=\mathrm{F}(S) \cup\{G\}$.

Proof. (1) Let $P$ be a prime ideal of $S$ with $P \varsubsetneqq M$. We will show that $P$ is strongly prime. Let $a+b \in P$ for $a, b \in G$. We may assume that $a \notin S$. Since $M$ is strongly prime, we have $b \in M$. Since $M \ni b=a+b-a$, we have $b-a \in M$. Then $2 b=b-a+a+b \in P$, and hence $b \in P$.

(2) There exists a valuation oversemigroup $V_{1}$ with maximal ideal $N$ such that $N \cap S=M$. Let $m \in M$, and let $w \in V_{1}$. Since $m=w+m-w$ and $-w \notin M$, we have $w+m \in M$. Hence $M$ is a prime ideal of $V_{1}$. Similarly, $M$ is a prime ideal of the valuation semigroup $V=\left(V_{1}\right)_{M}$. Hence $M$ is a maximal ideal of $\left(V_{1}\right)_{M}$.

(3) Let $V$ be a valuation oversemigroup of $S$ with maximal ideal $M$, and let $v$ be a valuation on $G$ belonging to $V$. Let $I \in \mathrm{F}^{\prime}(S)$. If $v(I)$ is bounded below, then $I \in \mathrm{F}(S)$. If $v(I)$ is not bounded below, then $I=G$.

Lemma 6. Assume that $S$ is a PVS with maximal ideal $M$ which is not a valuation semigroup.

(1) $M$ is not a principal ideal of $S$.

(2) $V=M^{-1}$ is a valuation oversemigroup with maximal ideal $M$. 
(3) $M$ is a divisorial ideal of $S$.

(4) Each prime ideal of $S$ is a prime ideal of $V$, and $\operatorname{dim}(V)=\operatorname{dim}(S)$.

(5) $M^{-1}=(M: M)$.

Proof. (1) Assume the contrary. We have $M=x+S$ for some $x \in M$. Let $W$ be a valuation oversemigroup of $S$ with maximal ideal $M$. Since $W+M=M$, we have $W=S$. Hence $S$ is a valuation semigroup; a contradiction.

(2) Let $a \in V$. Since $M$ is not a principal ideal of $S$, we have $a+M \subset M$. Hence $M$ is an ideal of $V$.

Let $x, y \in V$. Then $x+y+M \subset x+M \subset M$. Hence $x+y \in V$, and hence $V$ is an oversemigroup of $S$.

Let $z \in \mathrm{q}(S)-V$. We have $z+m \notin M$ for some $m \in M$. Since $m=z+m-z$, we have $-z \in M$. Hence $V$ is a valuation semigroup.

Let $\alpha \in V-M$. Since $m=-\alpha+m+\alpha$, we have $-\alpha+m \in M$. Hence $-\alpha \in V$. That is, $\alpha$ is a unit of $V$. Hence $M$ is a maximal ideal of $V$.

(3) If $M^{v} \supsetneqq M$, then $M^{v}=S$. It follows that $M^{-1}=S$, and hence $S$ is a valuation semigroup; a contradiction.

(4) Let $P$ be a prime ideal of $S$. It suffices to show that $V+P \subset P$. Let $x \in V$ and $p \in P$. If $x \in M$, then $x+p \in P$. If $x \notin M$, then $-x \in V-M$. Since $p=x+p-x$, we have $x+p \in P$.

(5) Suppose the contrary. There exists an element $x \in M^{-1}$ such that $x \notin(M: M)$. Then $x+M=S$, and hence $M$ is a principal ideal of $S$; a contradiction.

Lemma 7. Let $S$ be a PVS with maximal ideal $M$ which is not a valuation semigroup, let $V=M^{-1}$, let $H$ be the unit group of $S$, and let $L$ be the unit group of $V$.

(1) If $|L / H|=\infty$, or if $\operatorname{dim}(S)=\infty$, then $\left|\Sigma^{\prime}(S)\right|=\infty$.

(2) Assume that $|L / H|<\infty$. Let $T$ be an oversemigroup of $S$. Then, either $T \supset V$, or there exists a subgroup $K$ of $L$ with $K \supset H$ such that $T=K \cup M$.

Proof. (1) If $|L / H|=\infty$, there exists an infinite number of subgroups $K$ of $L$ containing $H$. Let $T=K \cup M$. Then $T$ is an oversemigroup of $S$.

(2) Assume that $T \not \subset V$. Choose $t \in T-V$, and let $\alpha \in L$. Then $-t \in M$ and $\alpha-t \in M$. It follows that $\alpha=t+(\alpha-t) \in T$, and that $T \supset V$.

Let $T$ be an oversemigroup of $S$ with $T \subset V$. Let $K=T-M$. Since $|L / H|<\infty$, each element $\alpha \in K$ is integral over $H$. Therefore $K$ is a sugbgroup of $L$ with $K \supset H$, and $T=K \cup M$.

Theorem 13. Let $S$ be a PVS which is not a valuation semigroup, let $M$ be the maximal ideal of $S$, and let $V=M^{-1}$. Let $\Sigma_{1}^{\prime}$ be the set of semistar operations $*$ on $S$ such that $S^{*} \supset V$, and let $\Sigma_{2}^{\prime}$ be the set of semistar operations * on $S$ such that $S^{*} \varsubsetneqq V$. Let $H$ be the unit group of $S$, and let $L$ be the unit group of $V$. Assume that $|L / H|<\infty$ and that $\operatorname{dim}(S)<\infty$. Let $H_{1}, \cdots, H_{l}$ be the subgroups $H^{\prime}$ of $L$ such that $L \supsetneqq H^{\prime} \supset H$, and let $S_{i}=H_{i} \cup M$ for each $i$.

(1) $\Sigma^{\prime}(S)=\Sigma_{1}^{\prime} \cup \Sigma_{2}^{\prime}$.

(2) $\left|\Sigma_{1}^{\prime}\right|=\left|\Sigma^{\prime}(V)\right|$.

(3) $\left|\Sigma_{2}^{\prime}\right|=\sum_{1}^{l}\left|\Sigma\left(S_{i}\right)\right|$. 
(4) $\left|\Sigma^{\prime}(V)\right|<\infty$, and $\left|\Sigma\left(S_{i}\right)\right|<\infty$ for each i.

Proof. (1) follows from Lemma 7 (2).

(3) Let $* \in \Sigma\left(S_{i}\right)$ for some $i$, let $*^{\prime}$ be an extension of $*$ to a semistar operation on $S_{i}$, and let $\mu(*)$ be the descent of $*^{\prime}$ to $S$. Then we have $S^{\mu(*)}=S_{i}$. Therefore $\Sigma\left(S_{i}\right) \cap \Sigma\left(S_{j}\right)=\emptyset$ for $i \neq j$, and $\mu(*) \in \Sigma_{2}^{\prime}$. Then $\mu$ is a bijection from $\cup \Sigma\left(S_{i}\right)$ onto $\Sigma_{2}^{\prime}$.

(4) Let $v$ be a valuatiuon on $G=\mathrm{q}(S)$ belonging to $V$, and let $\Gamma$ be the value group of $v$.

It suffices to show that $|\Sigma(S)|<\infty$. Thus let $C=L / H$, and let $\Pi$ be the set of nonempty subsets of $C$. Let $\Pi^{\Pi}$ be the set of mappings from $\Pi$ to $\Pi$. Then $\Pi^{\Pi}$ is a finite set. An element $c$ of $C$ canonically generates a fractional ideal $c+S$ of $S$. And an element $\sigma$ of $\Pi$ canonically generates a fractional ideal $\sigma+S$ of $S$.

Let $*$ be a star operation on $S$. Since $V=M^{-1}, V$ is a divisorial fractional ideal of $S$. Hence, for each $\sigma \in \Pi$, we have $(\sigma+S)^{*} \subset V^{*} \subset V^{v}=V$. Therefore $(\sigma+S)^{*}=\sigma_{1}+S$ for some $\sigma_{1} \in \Pi$. Then $\sigma_{1}$ is uniquely determined by $\sigma$. It follows that $*$ gives a mapping $\sigma \longmapsto \sigma_{1}$ from $\Pi$ to $\Pi$, that is, $*$ gives an element $F(*)$ of $\Pi^{\Pi}$.

Then $F$ is a maping from $\Sigma(S)$ to $\Pi^{\Pi}$. We will show that $F$ is an injective mapping.

Assume that star operations $*_{1}$ and $*_{2}$ of $S$ give the same element of $\Pi^{\Pi}$. We will show that $*_{1}=*_{2}$. Let $I$ be a fractional ideal of $S$. We must show that $I^{*_{1}}=I^{*_{2}}$.

There arise the following three cases: (i) $v(I)$ has a minimal element $v(x)$ with $x \in I$. (ii) $v(I)$ does not have a minimal element, and there exists $v(x)=\inf$ $\Gamma_{v}(I)$ with $x \in G$. (iii) There does not exist inf $\Gamma_{v}(I)$.

The case (i): We may assume that $I$ is a fractional ideal of $S$ with $I \ni 0$ such that $v(I)$ has the minimal element 0 . Then $I=\sigma+S$ for some $\sigma \in \Pi$. It folllows that $I^{*_{1}}=I^{*_{2}}$.

The case (ii): We may assume that $I$ is a fractional ideal of $S$ such that inf ${ }^{\Gamma} v(I)=0$. Then we have $I=M$. Hence $I=I^{v}$, and hence $I^{*_{1}}=I^{*_{2}}$.

The case (iii): Let $X=\{x \in G \mid v(x) \leq v(i)$ for each $i \in I\}$. Let $y \notin I$. Then $v(y)<v(i)$ for each $i \in I$. By the assumption, there exists $z \in X$ such that $v(y)<v(z)$. Therefore $y \notin \cap\{x+S \mid x \in X\}$. It follows that $I=\cap\{x+S \mid x \in X\}$, and hence $I=I^{v}$. Therefore $I^{*_{1}}=I^{*_{2}}$.

Proposition 8. Let $S$ be a PVS with maximal ideal $M$. The following conditions are equivalent.

(1) $\left|\Sigma^{\prime}(S)\right|<\infty$.

(2) $\operatorname{dim}(S)<\infty$, and $(M: M)$ is a finitely generated $S$-module.

Proof. $V=(M: M)$ is a valuation oversemigroup of $S$, and, if $S$ is a valuation semigroup, the assertion holds.

Thus assume that $S$ is not a valuation semigroup. Then $V=M^{-1}$ by Lemma 6 .

$(1) \Longrightarrow(2)$ : Then $V$ is a finitely generated semigroup over $S$, and $\bar{S}$ is a valuation semigroup (Lemma 1 ). Since $V$ and $\bar{S}$ have the common maximal ideal $M$, we have $V=\bar{S}$. It follows that $V$ is a finitely generated $S$-module. 
$(2) \Longrightarrow(1)$ : Let $H$ (resp. $L$ ) be the unit group of $S$ (resp. $V$ ). Then we have $|L / H|<\infty$. Theorem 13 implies that $\left|\Sigma^{\prime}(S)\right|<\infty$.

Theorem 14. Assume that $S$ and $\bar{S}$ have a common maximal ideal $M$. Let $H$ (resp. $\bar{H}$ ) be the group of units of $S$ (resp. $\bar{S}$ ). Then the following conditions are equivalent.

(1) $\left|\Sigma^{\prime}(S)\right|<\infty$.

(2) $\operatorname{dim}(S)<\infty, \bar{S}$ is a valuation semigroup, and $\bar{H} / H$ is a finite group.

Proof. (1) $\Longrightarrow(2)$ : If $|\bar{H} / H|=\infty$, there exists an infinite number of subgroups $K$ with $K \supset H$ of $\bar{H}$. Then $T=K \cup M$ is an oversemigroup of $S$. Then there arises an infinite number of semistar operations on $S$.

$(2) \Longrightarrow(1)$ : Then $S$ is a PVS, and $\bar{S}=(M: M)$. Proposition 8 implies $\left|\Sigma^{\prime}(S)\right|<\infty$.

Let $D$ be a domain, and let $P$ be a prime ideal of $D$. If $a b \in P$ implies either $a \in P$ or $b \in P$ for elements $a, b \in \mathrm{q}(D)$, then $P$ is called a strongly prime ideal of $D$. If each prime ideal of $D$ is strongly prime, then $D$ is called a pseudo-valuation domain (or a PVD).

Lemma 8. Let $M$ be a maximal ideal of $D$, and let $K=\mathrm{q}(D)$.

(1) If $D$ is a PVD, then $D$ is a local ring.

(2) If $D$ is a local ring with $M$ strongly prime. Then $D$ is a $P V D$.

(3) If $D$ is a PVD, then there exists a valuation overring $W$ of $D$ with maximal ideal $M$.

(4) If $D$ is a PVD, then $\mathrm{F}^{\prime}(D)=\mathrm{F}(D) \cup\{K\}$.

Proof. (1) Suppose the contrary. There exists a prime ideal $P$ of $D$ with $P \not \subset M$. Choose $a \in P-M$ and $b \in M-P$. Since $M \ni b=a \cdot \frac{b}{a}$, we have $\frac{b}{a} \in M$. Hence $b \in P$; a contradiction.

The following is a ring version of Lemma 6 .

Lemma 9. Let $D$ be a PVD with maximal ideal $M$ which is not a valuation ring.

(1) $M$ is not a principal ideal of $D$.

(2) $V=M^{-1}$ is a valuation ring with maximal ideal $M$.

(3) $M$ is a divisorial ideal of $D$.

(4) Each prime ideal of $D$ is a prime deal of $V$, and $\operatorname{dim}(V)=\operatorname{dim}(D)$.

(5) $M^{-1}=(M: M)$.

Lemma 10. Let $D$ be a $P V D$ with maximal ideal $M$ which is not a valuation ring. Let $V=M^{-1}$, let $K=V / M$, and let $k=D / M$.

(1) Let $T$ be an overring of $D$. Then either $T \supset V$ or $T \subset V$.

(2) Assume that $T \subset V$ be an overring of $D$, and that $T$ is integral over $D$. Then $T$ is a PVD with maximal ideal $M$.

(3) If $\left|\Sigma^{\prime}(D)\right|<\infty$, then $V$ is the integral closure of $D$, and $V$ is a finitely generated $D$-module.

(4) If $[K: k]=\infty$, or if $\operatorname{dim}(D)=\infty$, then $\left|\Sigma^{\prime}(D)\right|=\infty$. 
(5) There exists only a finite number of intermediate rings between $D$ and $V$ if and only if $K$ is a simple extension field of $k$ with $[K: k]<\infty$.

Proof. (1) Assume that $T \not \subset V$. Choose $t \in T-V$, and let $\alpha \in V$. Then $t^{-1} \in M$ and $\alpha t^{-1} \in M$. It follows that $\alpha=t\left(\alpha t^{-1}\right) \in T$, and hence $T \supset V$.

(2) Let $\alpha \in T-M$. Let $\alpha^{n}+a_{1} \alpha^{n-1}+\cdots+a_{0}=0$ be an integral equation of $\alpha$ over $D$ with minimal degree $n$. If $a_{0} \in M$, then $\alpha^{n-1}+a_{1} \alpha^{n-2}+\cdots+a_{1}=m$ belongs to $M$; a contradiction to the choice of $n$. Hence $a_{0} \notin M$. It follows that $\alpha$ is a unit of $T$, and that $M$ is the unique maximal ideal of $T$.

(3) $\bar{D}$ is a PVD with maximal ideal $M$ by (2). Then $\bar{D}$ is a valuation ring by Theorem 3 . It follows that $\bar{D}=V$. Since $V$ is a finitely generated overring of $D, V$ is a finitely generated $D$-module.

(4) If $[K: k]=\infty$, there exists an infinite number of intermediate rings $T$ between $D$ and $V$.

(5) If $[K: k]<\infty$, then $V$ is integral over $D$.

Let $k_{1}$ be a field, and let $k_{2}$ be an extension field of $k_{1}$. Then there exists only a finite number of intermediate fields between $k_{1}$ and $k_{2}$ if and only if $k_{2}$ is a simple extension of $k_{1}$ with finite degree.

Let $T$ be an overring of $D$, and let $*$ be a semistar operation on $D$. If we let $J^{\alpha(*)}=J^{*}$ for each $J \in \mathrm{F}^{\prime}(T)$, then $\alpha(*)$ is a semistar operation on $T$, and is called the ascent of $*$ to $T$.

Theorem 15. Let $D$ be a PVD which is not a valuation ring, let $M$ be a maximal ideal of $D$, and let $V=M^{-1}$. Assume that $\operatorname{dim}(D)<\infty$, and that $V / M$ is a simple extension field of $D / M$ with $[V / M: D / M]<\infty$. Let $\left\{D_{1}, \cdots, D_{l}\right\}$ be the set of overrings $T$ of $D$ such that $T \varsubsetneqq V$. Let $\Sigma_{1}^{\prime}$ be the set of semistar operations $*$ on $D$ such that $D^{*} \supset V$, and let $\Sigma_{2}^{\prime}$ be the set of semistar operations * on $D$ such that $D^{*} \varsubsetneqq V$.

(1) $\Sigma^{\prime}(D)=\Sigma_{1}^{\prime} \cup \Sigma_{2}^{\prime}$.

(2) $\left|\Sigma^{\prime}(V)\right|<\infty$.

(3) $\left|\Sigma_{1}^{\prime}\right|=\left|\Sigma^{\prime}(V)\right|$.

(4) There is a canonical bijection from the disjoint union $\cup_{1}^{l} \Sigma\left(D_{i}\right)$ onto $\Sigma_{2}^{\prime}$.

Proof. (1) follows from Lemma 10 (1).

(3) Let $* \in \Sigma^{\prime}(V)$, and let $\delta(*)$ be the descent of $*$ to $D$. Then $\delta(*) \in \Sigma_{1}^{\prime}$. The mapping $\delta$ from $\Sigma^{\prime}(V)$ to $\Sigma_{1}^{\prime}$ is injective.

Let $* \in \Sigma_{1}^{\prime}$, and let $\alpha(*)$ be the ascent of $*$ to $V$. Then we have $*=\delta(\alpha(*))$. Therefore $\left|\Sigma_{1}^{\prime}\right|=\left|\Sigma^{\prime}(V)\right|$.

(4) The proof is similar to that of Theorem $13(3)$.

Remark 3. In Theorem 15, if $[V / M: D / M]$ is either 2 or 3 , then $|\Sigma(D)|<\infty$.

Proof. Let $v$ be a valuation belonging to $V, \Gamma$ be the value group of $v$, and $K=V / M, k=D / M$, and asume that $[K: k]=3$ (The case where $[K: k]=2$ is similar).

Let $U, W$ be distinct 2-dimensional $k$-subspaces of $K$ containing $k$. Let $1, s$ (resp. $1, t)$ be a basis of $U$ (resp. $W$ ). Then $K=k+k s+k t$. Hence there exist 
elements $a_{1}, a_{2}, a_{3}$ of $k$ such that $s t=a_{1}+a_{2} s+a_{3} t$. Let $\alpha=t-a_{2}$. Then $\alpha s=a_{1}+a_{3} t \in W$. It follows that $W=\alpha U$.

Let $D \varsubsetneqq I_{0} \varsubsetneqq V$ be a fixed element of $\mathrm{F}(D)$. Assume that $D \varsubsetneqq I \varsubsetneqq V$ for $I \in$ $\mathrm{F}(D)$. Then we have $I / M=\bar{x}\left(I_{0} / M\right)$ and $I_{0} / M=\bar{y}(I / M)$ for some $x, y \in V$ with $\bar{x} \bar{y}=\overline{1}$. Then $x y$ is a unit of $D$. Since $I+M=I$, we have $x I_{0} \subset I$. Similarly, $y I \subset I_{0}$. Therefore $x I_{0}=I$ and $y I=I_{0}$.

Note that $M$ and $V$ are divisorial fractional ideals of $D$.

Let $*$ be a star operation on $D$. Then $I_{0}^{*}=I_{0}$ or $I_{0}^{*}=V$.

Let $I$ be a nonzero fractional ideal of $D$.

If there does not exist inf ${ }^{\Gamma}(v(I))$, then $I=I^{v}$.

Assume that there exists inf $\Gamma^{\Gamma}(v(I))=v(x)$ with $v(x) \notin v(I)$. Then we have $x^{-1} I=M$, and hence $I=I^{v}$.

Assume that there exists a minimal element $v\left(i_{0}\right)$ of $v(I)$ with $i_{0} \in I$. Since $D \subset i_{0}^{-1} I \subset V$, we have either $I=i_{0} D$ or $I=i_{0} V$ or $I=i I_{0}$ with $i \in I$.

The case where $I_{0}^{*}=I_{0}$ : Then $*=\mathrm{d}$.

The case where $I_{0}^{*}=V$ : We have $V=I_{0}^{*} \subset I_{0}^{v} \subset V$. Hence $*=\mathrm{v}$.

Proposition 9. Let $V$ be a valuation domain of the form $K+M$, where $K$ is a field and $M$ is the maximal ideal of $V$. Let $k$ be a subfield of $K$, and let $D=k+M$. Assume that $\operatorname{dim}(D)<\infty$, and $K$ is a simple extension of $k$ with finite degree. Then $|\Sigma(D)|$ need not be finite.

Proof. Let $k_{0}$ be a field with characteristic 0 , let $k=k_{0}\left(\pi^{4}\right)$, and let $K=k(\pi)$, where $\pi$ is an indeterminate over $k_{0}$. Let $V=K[[X]]$ be the ring of formal power series of an indeterminate $X$ over $K$, let $M$ be the maximal ideal of $V$, let $D=k+M$, let $v$ be the canonical $\mathbf{Z}$-valued valuation on $\mathrm{q}(D)$ belonging to $V$.

Fact 1. There exists an infinite number of 2-dimensional $k$-subspaces $U \supset k$ of $K$.

For, let $a, b$ be distinct elements of $k$, let $u_{1}=a \pi+\pi^{2}, u_{2}=b \pi+\pi^{2}$, and let $U_{1}=k+k u_{1}, U_{2}=k+k u_{2}$. Then $U_{1} \neq U_{2}$.

Fact 2. Let $a, b$ be elements of $k$ with $b \neq \pm a$, let $u_{1}=a \pi+\pi^{2}, u_{2}=b \pi+\pi^{2}$, and let $U_{1}=k+k u_{1}$, let $U_{2}=k+k u_{2}$. Then there exist no elements $\alpha \in K$ such that $\alpha U_{1}=U_{2}$.

For, the proof of Fact 1 shows that $U_{1} \neq U_{2}$. Suppose that there exists $\alpha \in K$ such that $\alpha U_{1}=U_{2}$. Then we have $\alpha=c_{0}+c_{1} u_{2}$ and $\alpha^{-1}=d_{0}+d_{1} u_{1}$ for some $c_{0}, c_{1}, d_{0}, d_{1} \in k$. Since $U_{1} \neq U_{2}$, we have $c_{1} \neq 0$ and $d_{1} \neq 0$. Seeing the coefficient of $\pi^{3}$ in the equation $\alpha \alpha^{-1}=\left(c_{0}+c_{1} b \pi+c_{1} \pi^{2}\right)\left(d_{0}+d_{1} a \pi+d_{1} \pi^{2}\right)$, we have $c_{1} d_{1}(a+b)=0$; a contradiction.

Fact 3. Let $I$ be a nonzero fractional ideal of $D$. Then there exist $x \in \mathrm{q}(D)$ and a $k$-subspace $U \supset k$ of $K$ such that $I=x U D$.

Moreover, let also $x U D=y W D$, where $W \supset k$ is a $k$-subspace of $K$ and $y \in$ $\mathrm{q}(D)$. Then we have $\operatorname{dim}(W)=\operatorname{dim}(U), W=\alpha U$ for some $\alpha \in K$, and $y=x u$ for some unit $u$ of $V$.

For, let $x$ be an element of $I$ such that $v(x)$ is the minimal element of $v(I)$. Let $U=x^{-1} I \cap K$. Then we have $x^{-1} I=U D$, and hence $I=x U D$. 
Fact 4. Let $U_{0} \supset k$ be a fixed 2-dimensional $k$-subspace of $K$. Let $U \supset k$ be any $k$-subspace of $K$.

If $U=k$, let $(U D)^{*}=D$.

If $U=\alpha U_{0}$ for some $\alpha \in K$, let $(U D)^{*}=V$.

If $\operatorname{dim}(U)=2$, and if $U \neq \alpha U_{0}$ for each $\alpha \in K$, let $(U D)^{*}=U D$.

If $\operatorname{dim}(U) \geq 3$, let $(U D)^{*}=V$.

Let $I=x U D$ be a nonzero fractional ideal of $D$, where $x \in \mathrm{q}(D)$ and $U \supset k$ is a $k$-subspace of $K$. Let $I^{*}=x(U D)^{*}$.

Then there is defined a unique mapping $*$ from $\mathrm{F}(D)$ to $\mathrm{F}(D)$.

For, let also $I=y W D$ for $y \in \mathrm{q}(D)$ and a $k$-subspce $W \supset k$ of $K$. We must show that $x(U D)^{*}=y(W D)^{*}$. We have $y=x u_{0}$ and $W=\alpha_{0} U$, where $u_{0}$ is a unit of $V$ and $\alpha_{0} \in K$.

The case where $U=k$ : Then $W=k$, and hence $x D=y D$. We have $x(U D)^{*}=x D$, and $y(W D)^{*}=y D$.

The case where $U=\alpha U_{0}$ for some $\alpha \in K$ : Then $W=\alpha_{0} \alpha U_{0}$. We have $x(U D)^{*}=x V$, and $y(W D)^{*}=y V=x u_{0} V=x V$.

The case where $\operatorname{dim}(U)=2$ and $U \neq \alpha U_{0}$ for each $\alpha \in K$ : Then $W \neq \alpha_{0} U_{0}$ for each $\alpha \in K$. We have $x(U D)^{*}=x U D$, and $y(W D)^{*}=y W D$.

The case where $\operatorname{dim}(U) \geq 3$ : Then $\operatorname{dim}(W) \geq 3$. We have $x(U D)^{*}=x V$, and $y(W D)^{*}=y V=x u_{0} V=x V$.

Let $*$ be the mapping from $\mathrm{F}(D)$ to $\mathrm{F}(D)$ defined in Fact 4.

Fact 5. Let $k \subset U \subset W$ be $k$-subspaces of $K$. Then $(U D)^{*} \subset(W D)^{*}$.

Fact 6. Let $x \in \mathrm{q}(D)-\{0\}$, and let $U, W$ be $k$-subspaces of $K$ containing $k$. If $U D \subset x W D$, then $(U D)^{*} \subset(x W D)^{*}$.

For, we may assume that $x=X^{-k} \alpha$, where $k$ is a nonnegative integer and $\alpha \in K$.

The case where $k>0$ : We have

$X^{k} \alpha^{-1}(U D)^{*} \subset X^{k} \alpha^{-1} V \subset M \subset D \subset(W D)^{*}$.

Hence $(U D)^{*} \subset(x W D)^{*}$.

The case where $k=0$ : Then $x=\alpha \in K$, and $U \subset \alpha W$. Fact 5 implies that $(U D)^{*} \subset x(W D)^{*}$.

Fact 7. The mapping $*$ from $\mathrm{F}(D)$ to $\mathrm{F}(D)$ is a star operation on $D$.

For, let $I \in \mathrm{F}(D)$. There exist $x \in \mathrm{q}(D)$ and a $k$-subspace $U \supset k$ of $K$ such that $I=x U D$. Then $I^{*}=x(U D)^{*}$. It follows that $I \subset I^{*}$.

The case where $I=x D$ with $x \in \mathrm{q}(D)$. We have $I^{*}=x D=I$.

Let $y \in \mathrm{q}(D)-\{0\}$. Since $y I=y x U D$, we have $(y I)^{*}=y x(U D)^{*}=y I^{*}$.

If $U=\alpha U_{0}$ for some $\alpha \in K$, then $I^{*}=x V$. We have $\left(I^{*}\right)^{*}=(x K D)^{*}=$ $x V=I^{*}$.

If $U$ is a 2-dimensional $k$-subspace of $K$ with $\alpha U_{0} \neq U$ for each $\alpha \in K$, then $I^{*}=x U D$. Hence $\left(I^{*}\right)^{*}=x U D=I^{*}$.

If $\operatorname{dim}(U) \geq 3$, then $I^{*}=x V$. Since $I^{*}=x K D$, we have $\left(I^{*}\right)^{*}=x V$, and hence $\left(I^{*}\right)^{*}=I^{*}$.

Assume that $I \subset J$ for $J \in \mathrm{F}(D)$. We have $J=y W D$, where $y \in \mathrm{q}(D)$ and $W \supset k$ is a $k$-subspace of $K$. Fact 6 implies that $I^{*} \subset J^{*}$. 
Let $U_{0} \supset k$ be a 2-dimensional $k$-subspace of $K$, and let $*\left(U_{0}\right)$ be the star operation on $D$ defined in Fact 7. Thus, for each 2-dimensional $k$-subspace $U \supset k$ of $K$, there is defined a star operation $*(U)$ on $D$. Let $U_{1}$ and $U_{2}$ be 2-dimensional $k$-subspaces of $K$ containing $k$ such that $U_{2} \neq \alpha U_{1}$ for each $\alpha \in K$. Then we have $\left(U_{1} D\right)^{*\left(U_{1}\right)}=V$ and $\left(U_{1} D\right)^{*\left(U_{2}\right)}=U_{1} D$. Hence $*\left(U_{1}\right) \neq *\left(U_{2}\right)$. Fact 2 implies that $|\Sigma(D)|=\infty$.

Remark 4. Let $D$ be a PVD with maximal ideal $M$ such that $\operatorname{dim}(D)<\infty$. If $V=(M: M)$ is a finitely generated $D$-module, $\left|\Sigma^{\prime}(D)\right|$ need not be finite.

Let $I$ be an ideal of $S$. If $a+b \in I$ and $b \notin I$ imply $n a \in I$ for some $n>0$ for elements $a, b \in \mathrm{q}(S)$, then $I$ is called a strongly primary ideal of $S$. If each prime ideal of $S$ is strongly primary, then $S$ is called an almost pseudo-valuation semigroup (or an APVS).

Let $I$ be an ideal of $S$. If $a+b \in I$ implies either $a \in S$ or $b \in S$ for elements $a, b \in \mathrm{q}(S)$, then $I$ is called a powerful ideal in $S$.

Lemma 11. Assume that $S$ has a strongly primary ideal $I$ with $I \varsubsetneqq S$.

(1) I is an ideal of $\bar{S}$.

(2) I is a powerful ideal in $\bar{S}$.

(3) $3 I$ is a powerful ideal in $S$.

(4) $V=(I: I)$ is a valuation oversemigroup of $S$.

(5) $I$ is a primary ideal in $V$, and the radical $M$ of $I$ in $V$ is the maximal ideal of $V$.

(6) $\mathrm{F}^{\prime}(S)=\mathrm{F}(S) \cup\{G\}$, where $G=\mathrm{q}(S)$.

Let $Q$ be a prime ideal of $S$ with $Q \varsubsetneqq I$. Then

(7) $Q \subset 3 I$, and $Q$ is a powerful ideal in $S$.

(8) $Q$ is strongly prime.

(9) $Q$ is a prime ideal of $V$.

Proof. (1) Suppose the contrary. There exists $x \in \bar{S}$ with $x+I \not \subset I$. We have $m x \in S$ for some $m>0$. Since $-x+x+I \subset I$, we have $-n x \in I$ for some $n>0$. Then we have

$$
0=n(m x)+m(-n x) \in S+I=I,
$$

and hence $I=S$; a contradiction.

(2) Let $x+y \in I$, and let $x \notin \bar{S}$. We have $n y \in I$ for some $n>0$, and hence $y \in \bar{S}$.

(3) Let $x+y \in 3 I$, and let $x \notin S$. Let $a, b \in I$. Since $(a+b-x)+(x-b) \in I$ and $x-b \notin S$, we have $n(a+b-x) \in I$ for some $n>0$. Then $a+b-x \in \bar{S}$. Hence $2 I \subset x+\bar{S}$. Then

$$
y=(-x)+(x+y) \in-x+3 I=(-x+I)+2 I \subset(-x+I)+x+\bar{S}=I+\bar{S} .
$$

Hence $y \in I$ by (1).

(4) Let $x \in G-V$. Then $\bar{S} \subset V$ by (1). Since $x-x+I=I$ and $x \notin \bar{S}$, we have $-x+I \subset I$. Therefore $-x \in V$, and hence $V$ is a valuation semigroup.

(5) $\quad I$ is a primary ideal of $V$ by the definition of $V$.

Suppose that $V_{M} \supsetneqq V$. Choose $x \in V_{M}$ with $x \notin V$. Since $-x+x+I \subset I$, we have $-n x \in I$ for some $n>0$. Then $0=-n x+n x \in I+V_{M}$, and hence 
$I+V_{M}=V_{M}$; a contradiction. Therefore $V=V_{M}$, and $M$ is the maximal ideal of $V$.

(6) Let $v$ be a valuation on $G$ belonging to $V$. Let $I^{\prime} \in \mathrm{F}^{\prime}(S)$.

The case where $v\left(I^{\prime}\right)$ is bounded below: There exists $x \in G$ such that $v(x)<$ $v(j)$ for each $j \in I^{\prime}$. Then $-x+I^{\prime} \subset V$. Hence $I^{\prime}$ is a fractional ideal of $S$.

The case where $v\left(I^{\prime}\right)$ is not bounded below: Let $x \in G$. There exists $y \in I^{\prime}$ such that $v(y)<v(x)$. Then $x=(x-y)+y \in V+y \subset I^{\prime}$. Hence $I^{\prime}=G$.

(7) Suppose that $Q \not \subset 3 I$. Choose $b \in Q-3 I$ and $a \in 3 I$. Since $a=$ $(2 a-b)+(b-a)$ and $b-a \notin S$, we have $2 a-b \in S$ by (3). Then we have $2 a \in b+S, 2 a \in Q, a \in Q, 3 I \subset Q$, and $I \subset Q$; a contradiction. Hence $Q \subset 3 I$.

Since $3 I$ is powerful in $S, Q$ is powerful in $S$.

(8) Let $x+y \in Q$. We may assume that either $x \notin S$ or $y \notin S$, say $x \notin S$. Since $Q$ is powerful in $S$, we have $y \in S$ and $2 x \notin Q$.

If $2 x \in S$; Since $2 x+2 y \in Q$, we have $2 y \in Q$, and hence $y \in Q$.

If $2 x \notin S$; Since $(2 y-x-y)+2 x \in Q$, we have $2 y-x-y \in S$. Then $2 y \in x+y+S \subset Q$, and hence $y \in Q$.

(9) Suppose the contrary. There exists an element $i \in I-Q$ such that $V+Q \ni i$. We have $i=x+q$ for some $x \in V$ and $q \in Q$. Since $i-x \in Q$, we have $-x \in Q$. It follows that $0=x-x \in V+I=I$; a contradiction.

Lemma 12. Let $S$ be an $A P V S$, let $P$ be the maximal ideal of $S$, and let $V=(P: P)$.

(1) If $S$ is not a valuation semigroup, then $V=P^{-1}$.

(2) The set of non-maximal prime ideals of $S$ coincides with the set of non-maximal prime ideals of $V$, and $\operatorname{dim}(V)=\operatorname{dim}(S)$.

(3) Let $T$ be an oversemigroup of $S$. Then either $T \supset V$ or $T \varsubsetneqq V$.

(4) Let $\Sigma_{1}^{\prime}=\left\{* \in \Sigma^{\prime}(S) \mid S^{*} \supset V\right\}$. Then there is a canonical bijection from $\Sigma^{\prime}(V)$ onto $\Sigma_{1}^{\prime}$.

(5) Let $\Sigma_{2}^{\prime}=\left\{* \in \Sigma^{\prime}(S) \mid S^{*} \varsubsetneqq V\right\}$. Then $\Sigma^{\prime}(S)=\Sigma_{1}^{\prime} \cup \Sigma_{2}^{\prime}$.

(6) If $\left|\Sigma^{\prime}(S)\right|<\infty$, then $\operatorname{dim}(S)<\infty$ and $V$ is a finitely generated $S$ module.

Proof. Lemma 11 implies that $V$ is a valuation oversemigroup of $S, P$ is a primary ideal of $V$, and the radical $M$ of $P$ in $V$ is the maximal ideal of $V$.

(1) Suppose the contrary. Choose an element $x \in P^{-1}-V$. Since $x+P \not \subset P$, we have $S=x+P$. It follows that $P=-x+S$, and $V=S$; a contradiction.

(2) If $P$ is not a maximal ideal of $V$, then $P$ is not a prime ideal of $V$. The assertion follows from Lemma 11 (9).

(3) Assume that $T \not \subset V$. There exists an element $t \in T-V$. Then $-t \in M$, and hence $-n t \in P$ for some $n>0$. Let $x \in V$. Then $x=n t+(x-n t) \in$ $T+P \subset T$, and hence $V \subset T$.

(6) Then $V$ is a finitely generated oversemigroup of $S$. By Lemma $1, \bar{S}$ is a valuation semigroup. Since $\bar{S} \subset V$, and $M$ is a maximal ideal of $\bar{S}$, we have $V=\bar{S}$. Therefore $V$ is a finitely generated $S$-module.

Lemma 13. Let $S$ be an APVS with dimension $<\infty$ which is not a PVS, $P$ be the maximal ideal of $S, V=(P: P)$ which is a finitely generated $S$-module, 
$M$ be the maximal ideal of $V, L$ be the unit group of $V, H$ be the unit group of $S, v$ be the valuation belonging to $V$, and $\Gamma$ be the value group of $v$.

(1) $V=\bar{S}$.

(2) $|L / H|<\infty$.

(3) There exist no $g_{i} \in L$ such that $V=S\left[g_{1}, \cdots, g_{l}\right]$.

(4) There exist $g_{i} \in L$ and $x_{0} \in M$ such that $V=S\left[g_{1}, \cdots, g_{l}, x_{0}\right]$.

(5) In (4), $v\left(x_{0}\right)$ is a minimal positive element of $\Gamma$.

(6) $\mathbf{Z} v\left(x_{0}\right)$ is a rank 1 convex subgroup of $\Gamma$.

(7) Let $m$ be a minimal positive integer $k$ such that $k x_{0} \in S$. Then $P=$ $\left\{x \in \mathrm{q}(S) \mid v(x) \geq m v\left(x_{0}\right)\right\}$.

Proof. (1) Since $V$ is a valuation oversemigroup of $S$ which is a finitely generated $S$-module, we have $V=\bar{S}$.

(2) Let $V=\left(S+x_{1}\right) \cup \cdots \cup\left(S+x_{n}\right)$. Let $\alpha \in L$. Then $\alpha=s+x_{i}$ for some $i$ with $s \in H$. Hence $|L / H| \leq n$.

(3) Suppose the contrary. For any $x \in M$, we have $x=s+\sum k_{i} g_{i}$ for $s \in S$ and $k_{i} \geq 0$. Then $s \in P$, hence $x \in P$. Therefore $P=M$, and hence $P$ is strongly prime; a contradiction.

(4) There exist $g_{1}, \cdots, g_{l} \in L$ and $x_{1}, \cdots, x_{m} \in M$ such that $V=S\left[g_{1}, \cdots\right.$, $\left.g_{l}, x_{1}, \cdots, x_{m}\right]$ with $m>0$. Assume that, for instance, $v\left(x_{2}\right)>v\left(x_{1}\right)$. We have $x_{2}-x_{1}=s+\sum k_{i} g_{i}+\sum k_{i}^{\prime} x_{i}$ for $s \in S$ and $k_{i} \geq 0, k_{i}^{\prime} \geq 0$. Hence $x_{2}=s+\sum k_{i} g_{i}+\left(1+k_{1}^{\prime}\right) x_{1}+\sum_{i=2}^{m} k_{i}^{\prime} x_{i}$. It follows that $k_{2}^{\prime}=0$, and $V=$ $S\left[g_{1}, \cdots, g_{l}, x_{1}, x_{3}, x_{4}, \cdots, x_{m}\right]$. Therefore we may assume that $v\left(x_{1}\right)=\cdots=$ $v\left(x_{m}\right)$. It follows that $V=S\left[g_{1}, \cdots, g_{l}, x_{1}\right]$.

(5) Suppose the contrary. There exists $x \in M$ such that $v(x)<v\left(x_{0}\right)$. Then $x_{0}-x=s+\sum k_{i} g_{i}+k x_{0}$. If $k>0$, then $x \in L$; a contradiction. If $k=0$, then $s \in P$. Then $x_{0}=x+s+\sum k_{i} g_{i} \in P$, and hence $V=S\left[g_{1}, \cdots, g_{l}\right]$; a contradiction.

(7) Assume that $\gamma \geq m v\left(x_{0}\right)$. Then $\gamma-m v\left(x_{0}\right)=v(x)$ for some $x \in V$, and $\gamma=v\left(m x_{0}+x\right) \in v(P)$.

A fractional ideal of $S$ is also called an $S$-fractional ideal, and a divisorial fractional ideal of $S$ is also called an $S$-divisorial fractional ideal.

Lemma 14. In Lemma 13, let $T$ be a subsemigroup of $V$ with $S \subset T \varsubsetneqq V$. Then $|\Sigma(T)|<\infty$.

Proof. Let $g_{1}, \cdots, g_{l}$ be a complete representative system of $L$ modulo $H$ with $g_{1}=0$. We have $V=S\left[g_{1}, \cdots, g_{l}, x_{0}\right]$ with $x_{0} \in M$. We may assume that $v\left(x_{0}\right)=1$, and that $\mathbf{Z}$ is a rank 1 convex subgroup of $\Gamma$.

(1) The set $\{I \mid I$ is a $T$-fractional ideal with $T \subset I \subset V\}$ is of finite.

For, $I \supset P$, and $I-P \subset\left\{g_{i}+h+k x_{0} \mid 1 \leq i \leq l, 0 \leq k<m\right.$, and $\left.h \in H\right\}$.

Let $\left\{I_{1}, \cdots, I_{a}\right\}$ be the set in (1).

(2) The set $\left\{I \mid I\right.$ is a $T$-fractional ideal with $\left.T \subset I \subset V-m x_{0}\right\}$ is of finite. For, $I \supset P$, and $I-P \subset\left\{g_{i}+h+k x_{0} \mid 1 \leq i \leq l,-m \leq k<m\right.$, and $\left.h \in H\right\}$. Let $\left\{J_{1}, \cdots, J_{b}\right\}$ be the set in (2).

(3) Let $I$ be a $T$-fractional ideal such that $\inf ^{\Gamma} v(I)$ does not exists. Then $I$ is a $T$-divisorial fractional ideal, that is, $I=I^{v_{T}}$. 
For, let $\{x \in \mathrm{q}(S) \mid x$ is a lower bound of $v(I)\}=\left\{x_{\lambda} \mid \lambda \in \Lambda\right\}$. Let $i \in I$, and let $x \in\left\{x_{\lambda} \mid \lambda \in \Lambda\right\}$. There exists $i_{1}, \cdots, i_{m} \in I$ such that $v\left(i_{m}\right)<v\left(i_{m-1}\right)<$ $\cdots<v\left(i_{1}\right)<v(i)$. Then we have $v\left(i_{m}\right)+m \leq v(i)$, and hence $v(i)-v(x)>m$. Therefore $i-x \in P$ and $i \in x+T$. It follows that $I \subset \cap_{\lambda}\left(x_{\lambda}+T\right)$. Conversely, let $y \in \cap\left(x_{\lambda}+T\right)$. Then the similar argument as above shows that $y \in I$. Therefore $I=\cap\left(x_{\lambda}+T\right)$.

(4) Let $I$ be a $T$-fractional ideal. Then either $v(I)$ has a minimal element or $\inf ^{\Gamma} v(I)$ does not exist.

For, suppose that $v(I)$ does not have a minimal element, and that there exists $x \in \mathrm{q}(S)$ with $v(x)=\inf ^{\Gamma} v(I)$. Then there exists $i \in I$ such that $v(x)<v(i)<$ $v(x)+1 ;$ a contradiction.

(5) The set $\left\{* \in \Sigma(T) \mid I_{i}^{*} \in\left\{J_{1}, \cdots, J_{b}\right\}\right.$ for each $\left.i\right\}$ is of finite.

For, let $*_{1}, *_{2}$ be elements of the set in (5), and assume that $I_{i}^{*_{1}}=I_{i}^{*_{2}}$ for each $i$. It suffices to show that $*_{1}=*_{2}$. Let $I$ be a $T$-fractional ideal.

The case where $v(I)$ has a minimal element $v(x)$ with $x \in I$ : Since $T \subset I-x \subset$ $V$, we have $I-x=I_{i}$ for some $i$. Since $I_{i}^{*_{1}}=I_{i}^{*_{2}}$, we have $I^{*_{1}}=I^{*_{2}}$.

The case where inf ${ }^{\Gamma} v(I)$ does not exist: Then $I=I^{v_{T}}$. Hence $I^{*_{1}}=I^{*_{2}}$.

Let $\left\{*_{1}, \cdots, *_{c}\right\}$ be the set in $(5)$.

(6) $\Sigma(T)=\left\{*_{1}, \cdots, *_{c}\right\}$.

For, let $* \in \Sigma(T)$. Since $m x_{0}+V \subset P$, we have $V^{*} \subset T-m x_{0}$. Since $T \subset I_{i}^{*} \subset V^{*} \subset V-m x_{0}$, we have $* \in\left\{*_{1}, \cdots, *_{c}\right\}$.

Theorem 16. Let $S$ be an $A P V S$, let $P$ be the maximal ideal of $S$, and let $V=(P: P)$. Then $\left|\Sigma^{\prime}(S)\right|<\infty$ if and only if $\operatorname{dim}(S)<\infty$ and $V$ is a finitely generated $S$-module.

Proof. The assetion holds for each PVS $S$ (Proposition 8). And the necessity of the assertion holds (Lemma $12(6)$ ).

Thus let $S$ be an APVS with dimension $<\infty$ which is not a PVS, $P$ be the maximal ideal of $S, V$ be a finitely generated $S$-module, $M$ be the maximal ideal of $V, L$ be the unit group of $V, H$ be the unit group of $S, v$ be the valuation belonging to $V$, and $\Gamma$ be the value group of $v$.

In Lemma 14, we have $V-S \subset\left\{g_{i}+h+k x_{0} \mid 1 \leq i \leq l, 0 \leq k<m\right.$, and $\left.h \in H\right\}$. Therefore the set $\{T \mid T$ is a subsemigroup of $V$ with $\bar{S} \subset T \varsubsetneqq V\}=\left\{T_{1}, \cdots, T_{\alpha}\right\}$ is a finite set. Let $\Sigma_{2}^{\prime}=\left\{* \in \Sigma^{\prime}(S) \mid S^{*} \varsubsetneqq V\right\}$. It suffices to show that $\left|\Sigma_{2}^{\prime}\right|<\infty$. By Lemma 14, we have $\left|\Sigma\left(T_{i}\right)\right|<\infty$, and $\left|\Sigma_{2}^{\prime}\right|=\sum\left|\Sigma\left(T_{i}\right)\right|<\infty$.

Let $I$ be an ideal of $D$. If $a b \in I$ and $b \notin I$ imply $a^{n} \in I$ for some $n>0$ for elements $a, b \in \mathrm{q}(D)$, then $I$ is called a strongly primary ideal of $D$. If $a b \in I$ implies either $a \in D$ or $b \in D$ for $a, b \in \mathrm{q}(D)$, then $I$ is called a powerful ideal in $D$.

If each prime ideal of $D$ is strongly primary, then $D$ is called an almost pseudovaluation domain (or an APVD).

Lemma 15. Assume that $D$ has a strongly primary ideal I with (0) $\varsubsetneqq I \varsubsetneqq D$.

(1) I is an ideal of $\bar{D}$.

(2) I is a powerful ideal in $\vec{D}$.

(3) $I^{3}$ is a powerful ideal in $D$. 
(4) $V=(I: I)$ is a valuation overring of $D$.

(5) $I$ is a primary ideal in $V$, and the radical of $I$ in $V$ is the maximal ideal of $V$.

(6) $\mathrm{F}^{\prime}(D)=\mathrm{F}(D) \cup\{\mathrm{q}(D)\}$.

(7) If $Q$ is a prime ideal of $D$ with $Q \varsubsetneqq I$. We have $Q \subset I^{3}$, and $Q$ is a powerful ideal in $D$.

(8) $Q$ is strongly prime.

(9) $Q$ is a prime ideal of $V$.

Lemma 16. Let $D$ be an $A P V D$, let $P$ be a maximal ideal of $D$, let $V=(P$ : $P)$, and let $M$ be the maximal ideal of $V$.

(1) $D$ is a local ring.

(2) If $D$ is not a valuation ring, then $V=P^{-1}$.

(3) The set of non-maximal prime ideals of $D$ coincides with the set of non-maximal prime ideals of $V$, and $\operatorname{dim}(V)=\operatorname{dim}(D)$.

(4) $\bar{D}$ is a PVD with maximal ideal $M$.

(5) Let $T$ be an overring of $D$. Then either $T \supset V$ or $T \varsubsetneqq V$.

(6) Let $\Sigma_{1}^{\prime}=\left\{* \in \Sigma^{\prime}(D) \mid D^{*} \supset V\right\}$. Then there exists a canonical bijection from $\Sigma^{\prime}(V)$ onto $\Sigma_{1}^{\prime}$.

(7) Let $\Sigma_{2}^{\prime}=\left\{* \in \Sigma^{\prime}(D) \mid D^{*} \varsubsetneqq V\right\}$. Then $\Sigma^{\prime}(D)=\Sigma_{1}^{\prime} \cup \Sigma_{2}^{\prime}$.

(8) If $\left|\Sigma^{\prime}(D)\right|<\infty$, then $\operatorname{dim}(D)<\infty, V=\bar{D}, V$ is a finitely generated $D$-module, and $V / M$ is a simple extension field of $D / P$ with $[V / M: D / P]<\infty$.

Proof. (1) Suppose the contrary. There exists a maximal ideal $Q$ of $D$ with $Q \not \subset P$. Choose $a \in Q-P$. Then $a \notin M$, that is, $a$ is a unit of $V$. Therefore $a^{-1} \in(P: P)$, and hence $P \subset a P \subset Q ;$ a contradiction.

(4) Suppose that $\bar{D}$ has a maximal ideal $N$ distinct from $M$. Choose $a \in$ $M-N$ and $b \in N-M$. We have $a^{n} \in P$ for some $n>0$. Since $b$ is a unit of $V$, we have $b^{-1} \in(P: P)$, and hence $P \subset b P \subset N$. It follows that $a \in N$; a contradiction. Then $\bar{D}$ is a PVD by Lemma $8(2)$.

(8) $\quad V$ is a finitely generated overring of $D$, and $\bar{D} \subset V$. Since $\left|\Sigma^{\prime}(\bar{D})\right|<\infty$, $\bar{D}$ is a valuation ring by Theorem 3 . Hence $V=\bar{D}$. Therefore $V$ is a finitely generated $D$-module.

Theorem 17. Let $D$ be an $A P V D$ which is not a $P V D$, let $P$ be a maximal ideal of $D$, and let $V=(P: P)$. Assume that $\operatorname{dim}(D)<\infty$, and let $\left\{T_{\lambda} \mid \lambda \in \Lambda\right\}$ be the set of overrings $T$ of $D$ with $T \varsubsetneqq V$. Let $\Sigma_{1}^{\prime}$ be the set of semistar operations $*$ on $D$ such that $D^{*} \supset V$, and let $\Sigma_{2}^{\prime}$ be the set of semistar operations * on $D$ such that $D^{*} \varsubsetneqq V$.

(1) $\Sigma^{\prime}(D)=\Sigma_{1}^{\prime} \cup \Sigma_{2}^{\prime}$.

(2) $\left|\Sigma^{\prime}(V)\right|<\infty$.

(3) $\left|\Sigma_{1}^{\prime}\right|=\left|\Sigma^{\prime}(V)\right|$.

(4) There is a canonical bijection from the disjoint union $\cup \Sigma\left(T_{\lambda}\right)$ onto $\Sigma_{2}^{\prime}$.

Proposition 10. Let $S$ be 1-dimensional such that the set $H$ of units of $S$ coincides with the set of units of $\bar{S}$. The following conditions are equivalent.

(1) $\left|\Sigma^{\prime}(S)\right|<\infty$. 
(2) $\bar{S}$ is a valuation semigroup on the quotient group $G$ of $S$, and $\bar{S}$ is a finitely generated $S$-module.

Proof. $\quad(2) \Longrightarrow(1)$ : We may assume that $S \varsubsetneqq \bar{S}$. Let $M$ (resp. $N$ ) be the maximal ideal of $S$ (resp. $V=\bar{S}$ ), let $v$ be the valuation on $G$ belonging to $V$, and let $\Gamma$ be the value group of $v$.

(i) Let $V=S\left[x_{1}, \cdots, x_{n}\right]$ with $x_{i} \notin S$ for each $i$. Let $v\left(x_{1}\right)=\min$ $\left\{v\left(x_{1}\right), \cdots, v\left(x_{n}\right)\right\}$. Then we have $v\left(x_{1}\right)=\min \{v(N)\}$.

For, suppose that there exists an element $y \in N$ such that $v(y)<v\left(x_{1}\right)$. We have $y=s+\sum k_{i} x_{i}$ with $s \in S$ and $k_{i} \geq 0$. By the choice of $y$, it follows that $k_{1}=\cdots=k_{n}=0$, and that $y=s \in M$. Put $y_{1}=x_{1}-y$. Since $y_{1} \in N$ and $v\left(y_{1}\right)<v\left(x_{1}\right)$, also it follows that $y_{1} \in M$. Then $x_{1}=y+y_{1} \in M$; a contradiction.

Thus $V$ is a discrete valuation semigroup, and we may assume that $\Gamma=\mathbf{Z}$ with $v\left(x_{1}\right)=1$.

(ii) There exists a positive integer $h$ such that $h+\mathbf{Z}_{0} \subset v(S)$.

(iii) The set $\{I \mid I$ is a fractional ideal of $S$ such that $S \subset I \subset V\}$ is of finite.

For, $V-S \subset\left\{k x_{1}+h \mid 0 \leq k<h\right.$ and $\left.h \in H\right\}$.

Let $\left\{J_{1}, \cdots, J_{a}\right\}$ be the set in (iii)

(iv) $\mathrm{F}^{\prime}(S)=\mathrm{F}(S) \cup\{G\}$.

(v) The set $\left\{* \in \Sigma^{\prime}(S) \mid J_{i}^{*} \in\left\{J_{1}, \cdots, J_{a}\right\}\right.$ for each $\left.i\right\}$ is of finite.

For, let $*_{1}, *_{2}$ be elements of the set in $(\mathrm{v})$, and assume that $J_{i}^{*_{1}}=J_{i}^{*_{2}}$ for each $i$. It suffices to show that $*_{1}=*_{2}$. Let $I$ be a fractional ideal of $S$. There exists an element $g \in G$ such that $S \subset g+I \subset V$. Hence $I=J_{i}-g$ for some $i$. Then we have $I^{*_{1}}=J_{i}^{*_{1}}-g=J_{i}^{*_{2}}-g=I^{*_{2}}$. It follows that $*_{1}=*_{2}$.

Let $\left\{*_{1}, \cdots, *_{b}\right\}$ be the set in $(\mathrm{v})$. Let $*$ be a semistar operation on $S$ which differs from the e-operation on $S$. Let $\alpha(*)$ be the ascent of $*$ to $V$. $V$ has exactly two semistar operations $\mathrm{d}_{V}^{\prime}$ and $\mathrm{e}_{V}$. Therefore $\alpha(*)$ is the $\mathrm{d}^{\prime}$-operation on $V$. Hence $S \subset J_{i}^{*} \subset V^{*}=V^{\alpha(*)}=V$ for each $i$, and hence $J_{i}^{*} \in\left\{J_{1}, \cdots, J_{a}\right\}$ for each $i$. It follows that $* \in\left\{*_{1}, \cdots, *_{b}\right\}$, and hence $\left|\Sigma^{\prime}(S)\right|<\infty$.

\section{REFERENCES}

[BH] A. Badawi and E. Houston, Powerful ideals, strongly primary ideals, almost pseudovaluation domains, and conducive domains, Comm. Alg. 30 (2002), 1591-1606.

[HH] J. Hedstrom and E. Houston, Pseudo-valuation domains, Pacific J. Math. 75 (1978), 137-147.

$[\mathrm{H}]$ W. Heinzer, Integral domains in which each non-zero ideal is divisorial, Mathematika 15 (1968), 164-170.

[M1] R. Matsuda, Note on the number of semistar-operations, Math. J. Ibaraki Univ. 31 (1999), 47-53.

[M2] R. Matsuda, Note on valuation rins and semistar-operations, Comm. Alg. 28 (2000), 2515-2519.

[M3] R. Matsuda, Note on the number of semistar-operations, IV, Sci. Math. Japon. 55 (2002), 345-347.

[M4] R. Matsuda, Note on the number of semistar-operations, V, Sci. Math. Japon. 57 (2003), 57-62.

[M5] R. Matsuda, Note on the number of semistar operations, VI, Internal. J. Commutative Rings 3 (2003). 
[M6] R. Matsuda, r-PVRs, r-APVSs and semistar operations, Math. J. Ibaralki Univ. 36 (2004), 33-43.

[M7] R. Matsuda, Note on the number of semistar perations, VII, Internal. J. Commutative Rings (to appear).

[M8] R. Matsuda, Note on almost pseudo-valuation domains, Proc. UAE Math-Day Conference, Nova Science, New York (to appear).

[MS] R. Matsuda and T. Sugatani, Semistar-operations on integral domains, II, Math. J. Toyama Univ. 18 (1995), 155-161. 